\title{
Domain Wall Dynamics in a Ferroelastic Spin Crossover Complex with Giant Magnetoelectric Coupling
}

Vibe Boel Jakobsen, ${ }^{\dagger}$ Elzbieta Trzop ${ }^{\ddagger}$ Emiel Dobbelaar, ${ }^{\dagger,}{ }^{\dagger}$ Laurence C. Gavin, ${ }^{\dagger}$ Shalinee Chikara, ${ }^{\delta, "}$ Xiaxin Ding, ${ }^{\prime \prime, \mathbb{a}}$ Minseong Lee, $\|$ Kane Esien, ${ }^{\perp}$ Helge Müller-Bunz, ${ }^{\dagger}$ Solveig Felton, ${ }^{\perp}$ Eric Collet, ${ }^{* \neq}$ Michael A. Carpenter, ${ }^{* \nabla}$ Vivien S. Zapf, ${ }^{*, \|}$ and Grace G. Morgan ${ }^{*, \dagger}$

${ }^{\dagger}$ School of Chemistry, University College Dublin, Belfield, Dublin 4, Ireland

‡ Univ Rennes, CNRS, IPR (Institut de Physique de Rennes) - UMR 6251, F-35000 Rennes, France

$\S$ Department of Physics, Auburn University Auburn, AL 36849, United States

II National High Magnetic Field Laboratory, Los Alamos National Laboratory, Los Alamos, New Mexico 87545, United States

${ }^{\perp}$ Centre for Nanostructured Media, School of Mathematics and Physics, Queen's University of Belfast, Belfast, BT7 1NN, Northern Ireland, United Kingdom

$\nabla^{\nabla}$ Department of Earth Sciences, University of Cambridge, Downing Street, Cambridge CB2 3EQ, England, United Kingdom

\section{Table of contents}

S1 Magnetometry measurements

S2 Single crystal X-ray diffraction

S3 Resonant ultrasound spectroscopy

S4 Magnetoelectric coupling

S5 References 
Table S1 Magnetic data for polycrystalline sample of complex 1 in cooling mode.

\begin{tabular}{|c|c|c|c|c|c|c|c|c|c|}
\hline \multicolumn{10}{|c|}{ Cooling mode } \\
\hline $\begin{array}{l}\text { Tempe- } \\
\text { rature } \\
\text { (K) }\end{array}$ & $\begin{array}{l}\chi_{\mathrm{M}} T \\
\left(\mathrm{~cm}^{3} \mathrm{~K}\right. \\
\left.\mathrm{mol}^{-1}\right)\end{array}$ & $\begin{array}{l}\text { Tempe- } \\
\text { rature } \\
\text { (K) }\end{array}$ & $\begin{array}{l}\chi_{\mathrm{M}} T \\
\left(\mathrm{~cm}^{3} \mathrm{~K}\right. \\
\left.\mathrm{mol}^{-1}\right)\end{array}$ & $\begin{array}{l}\text { Tempe- } \\
\text { rature } \\
\text { (K) }\end{array}$ & $\begin{array}{l}\chi_{\mathrm{M}} T \\
\left(\mathrm{~cm}^{3} \mathrm{~K}\right. \\
\left.\mathrm{mol}^{-1}\right)\end{array}$ & $\begin{array}{l}\text { Tempe- } \\
\text { rature } \\
\text { (K) }\end{array}$ & $\begin{array}{l}\chi_{\mathrm{M}} T \\
\left(\mathrm{~cm}^{3} \mathrm{~K}\right. \\
\left.\mathrm{mol}^{-1}\right)\end{array}$ & $\begin{array}{l}\text { Tempe- } \\
\text { rature } \\
\text { (K) }\end{array}$ & $\begin{array}{l}\chi_{\mathrm{M}} T \\
\left(\mathrm{~cm}^{3} \mathrm{~K}\right. \\
\left.\mathrm{mol}^{-1}\right)\end{array}$ \\
\hline 350.045 & 2.6348 & 273.605 & 2.6792 & 197.614 & 2.7462 & 121.705 & 2.3009 & 46.011 & 0.9935 \\
\hline 347.508 & 2.6312 & 271.603 & 2.6797 & 195.627 & 2.7495 & 119.710 & 2.2886 & 44.004 & 0.9909 \\
\hline 345.550 & 2.6057 & 269.596 & 2.6829 & 193.623 & 2.7512 & 117.701 & 2.2758 & 41.998 & 0.9874 \\
\hline 343.561 & 2.6090 & 267.583 & 2.6841 & 191.609 & 2.7529 & 115.708 & 2.2638 & 39.991 & 0.9841 \\
\hline 341.565 & 2.6111 & 265.586 & 2.6863 & 189.623 & 2.7550 & 113.706 & 2.2511 & 37.990 & 0.9802 \\
\hline 339.573 & 2.6136 & 263.614 & 2.6881 & 187.633 & 2.7564 & 111.721 & 2.2394 & 35.985 & 0.9755 \\
\hline 337.577 & 2.6158 & 261.566 & 2.6908 & 185.631 & 2.7590 & 109.721 & 2.2269 & 33.985 & 0.9704 \\
\hline 335.574 & 2.6182 & 259.583 & 2.6929 & 183.635 & 2.7594 & 107.727 & 2.2150 & 31.987 & 0.9657 \\
\hline 333.616 & 2.6210 & 257.593 & 2.6958 & 181.633 & 2.7625 & 105.734 & 2.2023 & 29.983 & 0.9583 \\
\hline 331.602 & 2.6225 & 255.591 & 2.6974 & 179.633 & 2.7637 & 103.734 & 2.1899 & 27.985 & 0.9487 \\
\hline 329.582 & 2.6248 & 253.597 & 2.6988 & 177.637 & 2.7656 & 101.737 & 2.1781 & 25.986 & 0.9373 \\
\hline 327.623 & 2.6277 & 251.599 & 2.7013 & 175.640 & 2.7682 & 99.748 & 2.1665 & 23.991 & 0.9229 \\
\hline 325.601 & 2.6300 & 249.585 & 2.7023 & 173.634 & 2.7695 & 97.743 & 2.1551 & 22.078 & 0.9052 \\
\hline 323.602 & 2.6304 & 247.599 & 2.7040 & 171.634 & 2.7714 & 95.777 & 2.1440 & 20.057 & 0.8822 \\
\hline 321.593 & 2.6334 & 245.593 & 2.7066 & 169.640 & 2.7722 & 93.777 & 2.1322 & 18.040 & 0.8522 \\
\hline 319.615 & 2.6362 & 243.588 & 2.7072 & 167.646 & 2.7742 & 91.768 & 2.1219 & 16.029 & 0.8139 \\
\hline 317.608 & 2.6377 & 241.615 & 2.7104 & 165.647 & 2.7753 & 89.767 & 2.1108 & 14.022 & 0.7643 \\
\hline 315.603 & 2.6395 & 239.595 & 2.7119 & 163.651 & 2.7770 & 87.809 & 2.1003 & 12.014 & 0.6989 \\
\hline 313.597 & 2.6423 & 237.589 & 2.7134 & 161.644 & 2.7777 & 85.829 & 2.0891 & 10.010 & 0.6172 \\
\hline 311.600 & 2.6439 & 235.600 & 2.7155 & 159.661 & 2.7790 & 83.830 & 2.0656 & 8.010 & 0.5161 \\
\hline 309.612 & 2.6444 & 233.599 & 2.7174 & 157.653 & 2.7789 & 82.063 & 1.5338 & 6.009 & 0.3976 \\
\hline 307.586 & 2.6472 & 231.596 & 2.7194 & 155.654 & 2.7808 & 80.054 & 1.1661 & 3.995 & 0.2697 \\
\hline 305.610 & 2.6487 & 229.590 & 2.7206 & 153.653 & 2.7811 & 78.058 & 1.1211 & & \\
\hline 303.605 & 2.6502 & 227.609 & 2.7213 & 151.659 & 2.7825 & 76.062 & 1.1000 & & \\
\hline 301.612 & 2.6524 & 225.591 & 2.7234 & 149.662 & 2.7832 & 74.062 & 1.0828 & & \\
\hline 299.594 & 2.6541 & 223.594 & 2.7252 & 147.669 & 2.7843 & 72.062 & 1.0686 & & \\
\hline 297.597 & 2.6555 & 221.602 & 2.7266 & 145.663 & 2.7854 & 70.060 & 1.0563 & & \\
\hline 295.609 & 2.6580 & 219.614 & 2.7280 & 143.657 & 2.7856 & 68.064 & 1.0462 & & \\
\hline 293.605 & 2.6595 & 217.605 & 2.7289 & 141.671 & 2.7869 & 66.085 & 1.0389 & & \\
\hline 291.593 & 2.6610 & 215.602 & 2.7303 & 139.675 & 2.7839 & 64.071 & 1.0314 & & \\
\hline 289.575 & 2.6631 & 213.602 & 2.7319 & 137.680 & 2.4306 & 62.071 & 1.0250 & & \\
\hline 287.611 & 2.6646 & 211.596 & 2.7334 & 135.669 & 2.3857 & 60.081 & 1.0198 & & \\
\hline 285.613 & 2.6667 & 209.607 & 2.7348 & 133.688 & 2.3732 & 58.091 & 1.0148 & & \\
\hline 283.603 & 2.6684 & 207.611 & 2.7361 & 131.679 & 2.3609 & 56.073 & 1.0104 & & \\
\hline 281.613 & 2.6711 & 205.612 & 2.7387 & 129.694 & 2.3485 & 54.074 & 1.0063 & & \\
\hline 279.602 & 2.6733 & 203.616 & 2.7392 & 127.690 & 2.3359 & 52.057 & 1.0028 & & \\
\hline 277.594 & 2.6743 & 201.618 & 2.7428 & 125.691 & 2.3239 & 50.040 & 0.9997 & & \\
\hline 275.595 & 2.6772 & 199.608 & 2.7456 & 123.699 & 2.3134 & 48.015 & 0.9968 & & \\
\hline
\end{tabular}


Table S2 Magnetic data for polycrystalline sample of complex $\mathbf{1}$ in warming mode.

\begin{tabular}{|c|c|c|c|c|c|c|c|c|c|}
\hline \multicolumn{10}{|c|}{ Warming mode } \\
\hline $\begin{array}{l}\text { Tempe- } \\
\text { rature } \\
\text { (K) }\end{array}$ & $\begin{array}{l}\chi_{\mathrm{M}} T \\
\left(\mathrm{~cm}^{3} \mathrm{~K}\right. \\
\left.\mathrm{mol}^{-1}\right)\end{array}$ & $\begin{array}{l}\text { Tempe- } \\
\text { rature } \\
\text { (K) }\end{array}$ & $\begin{array}{l}\chi_{\mathrm{M}} T \\
\left(\mathrm{~cm}^{3} \mathrm{~K}\right. \\
\left.\mathrm{mol}^{-1}\right)\end{array}$ & $\begin{array}{l}\text { Tempe- } \\
\text { rature } \\
\text { (K) }\end{array}$ & $\begin{array}{l}\chi_{\mathrm{M}} T \\
\left(\mathrm{~cm}^{3} \mathrm{~K}\right. \\
\left.\mathrm{mol}^{-1}\right)\end{array}$ & $\begin{array}{l}\text { Tempe- } \\
\text { rature } \\
\text { (K) }\end{array}$ & $\begin{array}{l}\chi_{\mathrm{M}} T \\
\left(\mathrm{~cm}^{3} \mathrm{~K}\right. \\
\left.\mathrm{mol}^{-1}\right)\end{array}$ & $\begin{array}{l}\text { Tempe- } \\
\text { rature } \\
\text { (K) }\end{array}$ & $\begin{array}{l}\chi_{\mathrm{M}} T \\
\left(\mathrm{~cm}^{3} \mathrm{~K}\right. \\
\left.\mathrm{mol}^{-1}\right)\end{array}$ \\
\hline 3.997 & 0.2698 & 80.124 & 1.1388 & 156.246 & 2.7953 & 232.308 & 2.7220 & 308.351 & 2.6449 \\
\hline 6.003 & 0.3973 & 82.120 & 1.1693 & 158.258 & 2.7939 & 234.303 & 2.7215 & 310.343 & 2.6431 \\
\hline 8.004 & 0.5159 & 84.129 & 1.2229 & 160.265 & 2.7932 & 236.305 & 2.7202 & 312.353 & 2.6409 \\
\hline 10.001 & 0.6175 & 86.134 & 1.3806 & 162.261 & 2.7930 & 238.307 & 2.7180 & 314.368 & 2.6407 \\
\hline 11.998 & 0.6986 & 88.152 & 1.8192 & 164.257 & 2.7920 & 240.315 & 2.7162 & 316.347 & 2.6381 \\
\hline 14.008 & 0.7636 & 90.148 & 2.0491 & 166.265 & 2.7904 & 242.320 & 2.7134 & 318.367 & 2.6363 \\
\hline 15.989 & 0.8130 & 92.157 & 2.1065 & 168.267 & 2.7896 & 244.320 & 2.7120 & 320.355 & 2.6333 \\
\hline 17.989 & 0.8510 & 94.167 & 2.1311 & 170.266 & 2.7883 & 246.317 & 2.7094 & 322.344 & 2.6320 \\
\hline 20.009 & 0.8812 & 96.166 & 2.1460 & 172.271 & 2.7876 & 248.308 & 2.7078 & 324.349 & 2.6296 \\
\hline 21.994 & 0.9043 & 98.161 & 2.1583 & 174.279 & 2.7853 & 250.321 & 2.7053 & 326.345 & 2.6275 \\
\hline 23.995 & 0.9225 & 100.173 & 2.1708 & 176.276 & 2.7841 & 252.316 & 2.7027 & 328.345 & 2.6249 \\
\hline 26.000 & 0.9370 & 102.181 & 2.1832 & 178.278 & 2.7822 & 254.323 & 2.7000 & 330.337 & 2.6228 \\
\hline 28.000 & 0.9486 & 104.180 & 2.1957 & 180.275 & 2.7813 & 256.326 & 2.6976 & 332.341 & 2.6225 \\
\hline 29.999 & 0.9577 & 106.192 & 2.2090 & 182.276 & 2.7786 & 258.326 & 2.6955 & 334.324 & 2.6210 \\
\hline 32.000 & 0.9653 & 108.183 & 2.2208 & 184.275 & 2.7766 & 260.320 & 2.6941 & 336.339 & 2.6174 \\
\hline 34.000 & 0.9715 & 110.194 & 2.2335 & 186.281 & 2.7747 & 262.323 & 2.6924 & 338.346 & 2.6166 \\
\hline 36.001 & 0.9767 & 112.189 & 2.2466 & 188.285 & 2.7726 & 264.351 & 2.6900 & 340.342 & 2.6145 \\
\hline 38.002 & 0.9811 & 114.205 & 2.2588 & 190.285 & 2.7694 & 266.333 & 2.6872 & 342.356 & 2.6133 \\
\hline 40.005 & 0.9851 & 116.193 & 2.2714 & 192.288 & 2.7679 & 268.323 & 2.6854 & 344.347 & 2.6110 \\
\hline 42.007 & 0.9884 & 118.198 & 2.2838 & 194.293 & 2.7665 & 270.322 & 2.6826 & 346.333 & 2.6100 \\
\hline 44.008 & 0.9916 & 120.191 & 2.2964 & 196.292 & 2.7645 & 272.325 & 2.6812 & 348.342 & 2.6087 \\
\hline 46.012 & 0.9948 & 122.240 & 2.3097 & 198.279 & 2.7622 & 274.326 & 2.6797 & 350.334 & 2.6074 \\
\hline 48.016 & 0.9976 & 124.238 & 2.3222 & 200.292 & 2.7600 & 276.331 & 2.6766 & & \\
\hline 50.030 & 1.0005 & 126.211 & 2.3339 & 202.293 & 2.7589 & 278.319 & 2.6750 & & \\
\hline 52.036 & 1.0034 & 128.200 & 2.3452 & 204.306 & 2.7561 & 280.334 & 2.6740 & & \\
\hline 54.047 & 1.0066 & 130.202 & 2.3573 & 206.292 & 2.7548 & 282.349 & 2.6714 & & \\
\hline 56.052 & 1.0100 & 132.210 & 2.3689 & 208.297 & 2.7521 & 284.326 & 2.6676 & & \\
\hline 58.055 & 1.0144 & 134.210 & 2.3817 & 210.299 & 2.7498 & 286.325 & 2.6658 & & \\
\hline 60.061 & 1.0185 & 136.204 & 2.3928 & 212.305 & 2.7481 & 288.329 & 2.6647 & & \\
\hline 62.066 & 1.0239 & 138.237 & 2.4070 & 214.303 & 2.7466 & 290.338 & 2.6619 & & \\
\hline 64.075 & 1.0300 & 140.227 & 2.5091 & 216.297 & 2.7437 & 292.325 & 2.6611 & & \\
\hline 66.081 & 1.0373 & 142.227 & 2.8015 & 218.303 & 2.7423 & 294.329 & 2.6590 & & \\
\hline 68.080 & 1.0455 & 144.235 & 2.7999 & 220.310 & 2.7394 & 296.333 & 2.6570 & & \\
\hline 70.094 & 1.0555 & 146.235 & 2.7976 & 222.304 & 2.7370 & 298.335 & 2.6557 & & \\
\hline 72.094 & 1.0671 & 148.254 & 2.7985 & 224.305 & 2.7352 & 300.343 & 2.6543 & & \\
\hline 74.107 & 1.0811 & 150.255 & 2.7973 & 226.313 & 2.7355 & 302.334 & 2.6527 & & \\
\hline 76.103 & 1.0966 & 152.266 & 2.7966 & 228.306 & 2.7318 & 304.330 & 2.6496 & & \\
\hline 78.106 & 1.1160 & 154.216 & 2.7948 & 230.318 & 2.7290 & 306.345 & 2.6473 & & \\
\hline
\end{tabular}




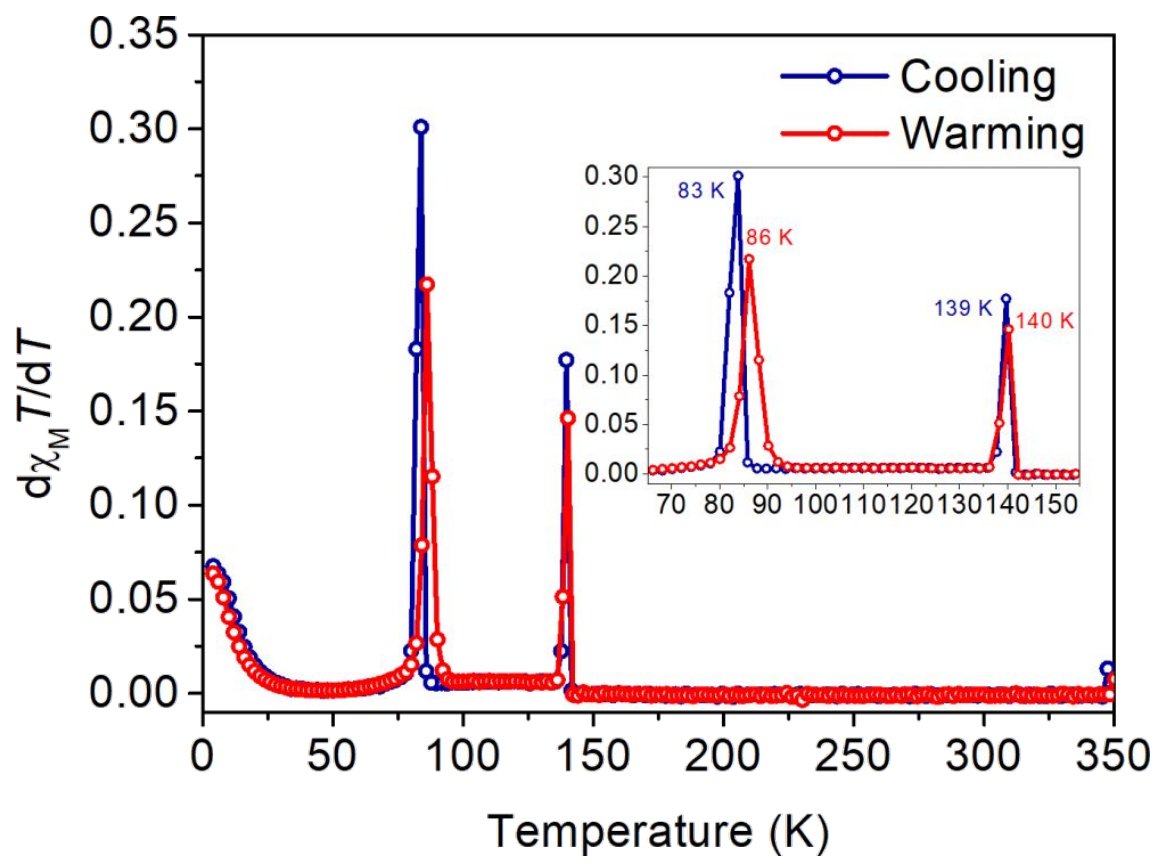

Figure S1 Derivative of $\chi_{M} T$ versus temperature, $T$ for complex 1 showing discontinuities. The inset shows $\mathrm{d} \chi_{\mathrm{M}} T / T$ maximum values at $83 \mathrm{~K}$ (cooling) and $86 \mathrm{~K}$ (heating) in the lower hysteresis region and $139 \mathrm{~K}$ (cooling) and $140 \mathrm{~K}$ (heating) in the upper hysteresis window.

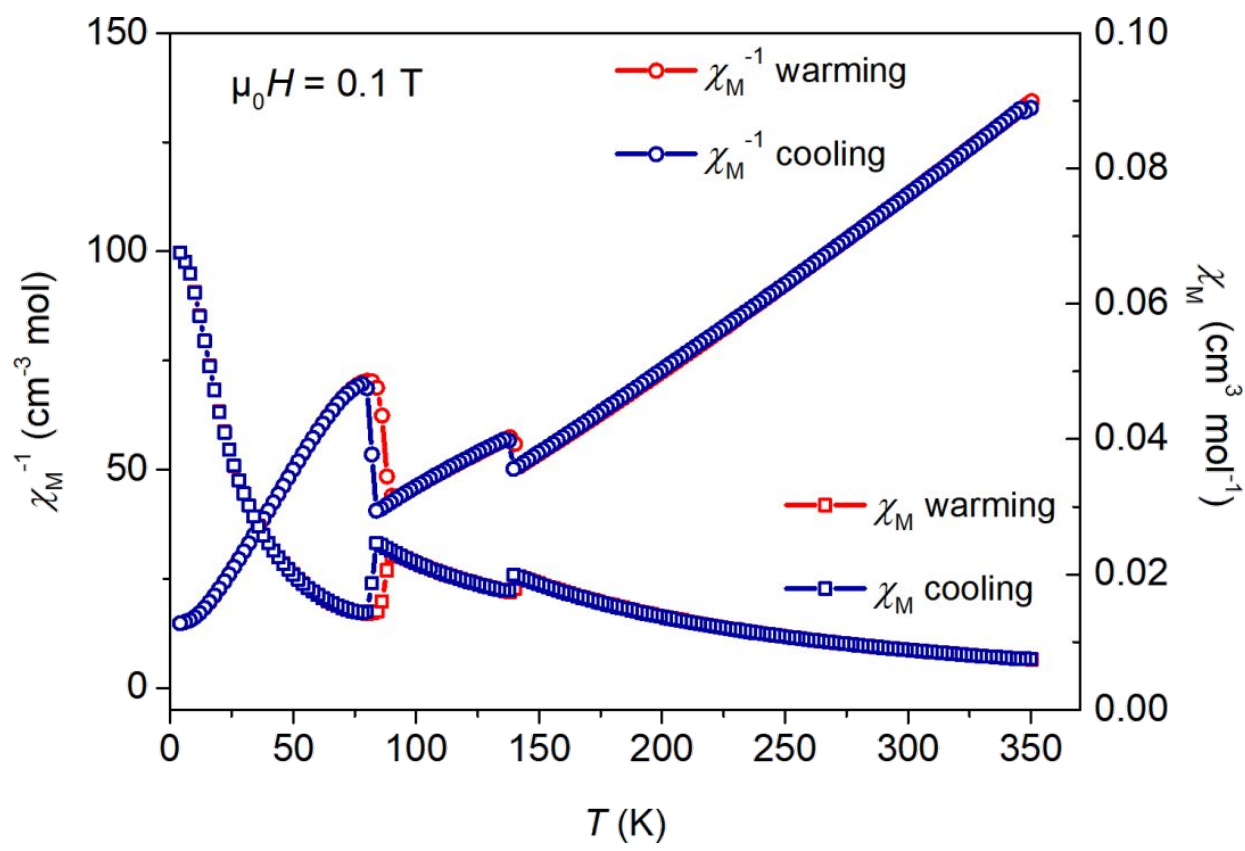

Figure S2 Temperature dependence of $\chi_{\mathrm{M}}^{-1}$ (left axis) and $\chi_{\mathrm{M}}$ (right axis) in warming and cooling modes for complex 1. 


\section{S2 Single crystal X-ray diffraction}

S2.1 Crystal and structure refinement data

Table S3 Crystallographic data for complex 1 at $10 \mathrm{~K}, 82 \mathrm{~K}, 115 \mathrm{~K}, 150 \mathrm{~K}$ and $250 \mathrm{~K}$.

\begin{tabular}{|c|c|c|c|c|c|}
\hline & $1,10 \mathrm{~K}$ & $1,82 \mathrm{~K}$ & $\mathbf{1}, 115 \mathrm{~K}$ & $1,150 \mathrm{~K}$ & $1,250 \mathrm{~K}$ \\
\hline CCDC number & 2100801 & 2100800 & 2100799 & 2100798 & 2100797 \\
\hline Empirical formula & $\mathrm{C}_{46} \mathrm{H}_{44} \mathrm{BCl}_{4} \mathrm{MnN}_{4} \mathrm{O}_{2}$ & $\mathrm{C}_{46} \mathrm{H}_{44} \mathrm{BCl}_{4} \mathrm{MnN}_{4} \mathrm{O}_{2}$ & $\mathrm{C}_{46} \mathrm{H}_{44} \mathrm{BCl}_{4} \mathrm{MnN}_{4} \mathrm{O}_{2}$ & $\mathrm{C}_{46} \mathrm{H}_{44} \mathrm{BCl}_{4} \mathrm{MnN}_{4} \mathrm{O}_{2}$ & $\mathrm{C}_{46} \mathrm{H}_{44} \mathrm{BCl}_{4} \mathrm{MnN}_{4} \mathrm{O}_{2}$ \\
\hline \multirow{2}{*}{ Molecular formula } & {$\left[\mathrm{C}_{22} \mathrm{H}_{24} \mathrm{~N}_{4} \mathrm{O}_{2} \mathrm{MnCl}_{4}\right]^{+}$} & {$\left[\mathrm{C}_{22} \mathrm{H}_{24} \mathrm{~N}_{4} \mathrm{O}_{2} \mathrm{MnCl}_{4}\right]^{+}$} & {$\left[\mathrm{C}_{22} \mathrm{H}_{24} \mathrm{~N}_{4} \mathrm{O}_{2} \mathrm{MnCl}_{4}\right]^{+}$} & {$\left[\mathrm{C}_{22} \mathrm{H}_{24} \mathrm{~N}_{4} \mathrm{O}_{2} \mathrm{MnCl}_{4}\right]^{+}$} & {$\left[\mathrm{C}_{22} \mathrm{H}_{24} \mathrm{~N}_{4} \mathrm{O}_{2} \mathrm{MnCl}_{4}\right]^{+}$} \\
\hline & {$\left[\mathrm{C}_{24} \mathrm{H}_{20} \mathrm{~B}\right]^{-}$} & {$\left[\mathrm{C}_{24} \mathrm{H}_{20} \mathrm{~B}\right]^{-}$} & {$\left[\mathrm{C}_{24} \mathrm{H}_{20} \mathrm{~B}\right]^{-}$} & {$\left[\mathrm{C}_{24} \mathrm{H}_{20} \mathrm{~B}\right]^{-}$} & {$\left[\mathrm{C}_{24} \mathrm{H}_{20} \mathrm{~B}\right]^{-}$} \\
\hline Formula weight & 892.40 & 892.40 & 892.40 & 892.40 & 892.40 \\
\hline Wavelength & 0.71073 & 0.71073 & 0.71073 & 0.71073 & 0.71073 \\
\hline Crystal system & Triclinic & Triclinic & Triclinic & Monoclinic & Monoclinic \\
\hline Space group & P1 & P1 & P1 & $\mathrm{Pc}$ & $\mathrm{Cc}$ \\
\hline $\mathrm{a}(\AA ̊)$ & $14.2889(4)$ & $14.4026(7)$ & $14.2241(4)$ & $14.7537(3)$ & $14.8473(5)$ \\
\hline$b(\AA)$ & $13.2527(7)$ & $13.3973(7)$ & $22.9492(6)$ & $22.6797(3)$ & $22.6658(5)$ \\
\hline$c(\AA ̊)$ & $13.8907(8)$ & 13.9646(7) & $13.4248(4)$ & $14.2596(3)$ & $14.4182(5)$ \\
\hline$\alpha\left({ }^{\circ}\right)$ & $117.134(6)$ & $117.044(5)$ & $94.753(2)$ & 90 & 90 \\
\hline$\beta\left({ }^{\circ}\right)$ & $104.637(4)$ & $104.573(4)$ & $103.722(2)$ & 117.133(3) & $117.027(4)$ \\
\hline$\gamma\left({ }^{\circ}\right)$ & $104.078(4)$ & $104.062(4)$ & $80.125(2)$ & 90 & 90 \\
\hline Volume $\left(\AA^{3}\right)$ & 2059.3(2) & $2113.7(2)$ & $4190.1(2)$ & $4246.30(17)$ & $4322.2(3)$ \\
\hline Z & 2 & 2 & 4 & 4 & 4 \\
\hline $\begin{array}{l}\text { Density (calculated) } \\
\left(\mathrm{mg} / \mathrm{m}^{3}\right)\end{array}$ & 1.439 & 1.402 & 1.415 & 1.396 & 1.371 \\
\hline $\begin{array}{l}\text { Absorption coefficient } \\
\left(\mathrm{mm}^{-1}\right)\end{array}$ & 0.625 & 0.609 & 0.615 & 0.607 & 0.596 \\
\hline Crystal size $\left(\mathrm{mm}^{3}\right)$ & $0.243 \times 0.151 \times 0.124$ & $0.298 \times 0.258 \times 0.133$ & $0.328 \times 0.235 \times 0.132$ & $0.334 \times 0.181 \times 0.152$ & $0.334 \times 0.181 \times 0.152$ \\
\hline Goodness-of-fit on $\mathrm{F}^{2}$ & 1.004 & 0.942 & 0.800 & 1.014 & 1.032 \\
\hline Final $R$ indices [ $1>2 \operatorname{sigma}(I)]$ & $R_{1}=0.0637, w R_{2}=$ & $R_{1}=0.0529, w R_{2}=$ & $R_{1}=0.0389, w R_{2}=$ & $R_{1}=0.0379, w R_{2}=$ & $R_{1}=0.0388, w R_{2}=$ \\
\hline 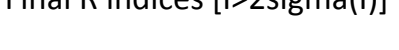 & 0.1462 & 0.1145 & 0.0692 & 00746 & 0.0781 \\
\hline $\begin{array}{l}\text { Absolute structure } \\
\text { parameter }\end{array}$ & $-0.16(3)$ & $0.042(17)$ & $0.023(10)$ & $-0.007(6)$ & $0.008(9)$ \\
\hline
\end{tabular}


Table S4 Selected bond lengths $(\AA)$ and angles $\left({ }^{\circ}\right)$ for complex 1 .

\begin{tabular}{|c|c|c|c|c|c|}
\hline & $1(10 \mathrm{~K})$ & $1(82 \mathrm{~K})$ & 1 (115 K) & $1(150 \mathrm{~K})$ & $1(250 \mathrm{~K})$ \\
\hline \multicolumn{6}{|c|}{ Bond lengths $(\AA ̊)$} \\
\hline Mn1-O1 $1_{\text {phen }}$ & $1.864(10)$ & $1.883(5)$ & $1.871(4)$ & $1.871(2)$ & $1.869(3)$ \\
\hline Mn1-O2 $2_{\text {phen }}$ & $1.883(10)$ & $1.897(5)$ & $1.881(4)$ & $1.872(2)$ & $1.872(3)$ \\
\hline $\mathrm{Mn} 1-\mathrm{N} 1_{\text {imine }}$ & $2.001(12)$ & $2.011(6)$ & $2.017(5)$ & $2.125(3)$ & $2.115(3)$ \\
\hline Mn1-N2 amine & $2.067(11)$ & $2.102(6)$ & $2.120(5)$ & $2.217(3)$ & $2.225(3)$ \\
\hline Mn1-N3 amine & $2.040(12)$ & $2.072(6)$ & $2.073(5)$ & $2.240(3)$ & $2.216(3)$ \\
\hline Mn1-N4 ${ }_{\text {imine }}$ & $1.967(11)$ & $2.007(7)$ & $2.013(6)$ & $2.079(3)$ & $2.088(3)$ \\
\hline Mn2-O3 $3_{\text {phen }}$ & $1.876(10)$ & $1.880(5)$ & $1.874(4)$ & $1.866(2)$ & \\
\hline Mn2-O4 phen & $1.877(10)$ & $1.902(5)$ & $1.880(4)$ & $1.875(2)$ & \\
\hline Mn2-N5 $5_{\text {imine }}$ & $1.987(12)$ & $2.001(7)$ & $2.034(5)$ & $2.107(3)$ & \\
\hline Mn2-N6 amine & $2.042(12)$ & $2.068(6)$ & $2.141(6)$ & $2.234(3)$ & \\
\hline Mn2-N7 amine & $2.089(11)$ & $2.096(6)$ & $2.107(6)$ & $2.204(3)$ & \\
\hline $\mathrm{Mn} 2-\mathrm{N} 8_{\text {imine }}$ & $1.999(12)$ & $2.020(6)$ & $2.027(6)$ & $2.090(3)$ & \\
\hline Mn3-O5 phen & & & $1.864(4)$ & & \\
\hline Mn3-06 & & & $1.875(4)$ & & \\
\hline Mn3-N9 $9_{\text {imine }}$ & & & $2.106(5)$ & & \\
\hline Mn3-N10 amine & & & $2.242(5)$ & & \\
\hline Mn3-N11 $1_{\text {amine }}$ & & & $2.206(5)$ & & \\
\hline Mn3-N12 imine & & & $2.081(5)$ & & \\
\hline Mn4-O7phen & & & $1.864(4)$ & & \\
\hline Mn4-08 $8_{\text {phen }}$ & & & $1.876(4)$ & & \\
\hline Mn4-N13 ${ }_{\text {imine }}$ & & & $2.101(6)$ & & \\
\hline Mn4-N14 amine & & & $2.239(7)$ & & \\
\hline Mn4-N15 amine & & & $2.229(7)$ & & \\
\hline Mn4-N16 imine & & & $2.091(5)$ & & \\
\hline \multicolumn{6}{|c|}{ Bond angles $\left({ }^{\circ}\right)$} \\
\hline O1-Mn1-O2 & $176.0(4)$ & $176.0(2)$ & 176.74(19) & $177.03(11)$ & $176.74(13)$ \\
\hline O1-Mn1-N1 & $89.7(4)$ & $89.7(2)$ & $89.1(2)$ & $87.36(11)$ & $87.52(12)$ \\
\hline O1-Mn1-N2 & $94.1(4)$ & $93.4(2)$ & $93.0(2)$ & $93.84(12)$ & $93.57(13)$ \\
\hline O1-Mn1-N3 & $85.0(4)$ & $85.2(2)$ & $85.2(2)$ & $85.03(12)$ & $85.97(13)$ \\
\hline O1-Mn1-N4 & $89.4(4)$ & $89.7(2)$ & $90.4(2)$ & $90.04(11)$ & $90.15(12)$ \\
\hline O2-Mn1-N1 & $94.1(4)$ & $94.1(2)$ & $94.01(19)$ & $92.78(11)$ & $92.96(12)$ \\
\hline O2-Mn1-N2 & $87.5(4)$ & $88.2(2)$ & $88.17(19)$ & $89.12(11)$ & $89.68(13)$ \\
\hline O2-Mn1-N3 & $91.5(4)$ & $91.3(2)$ & $91.9(2)$ & $95.81(12)$ & $94.58(14)$ \\
\hline O2-Mn1-N4 & $88.6(4)$ & $88.3(2)$ & $88.0(2)$ & $87.18(11)$ & $86.67(12)$ \\
\hline N1-Mn1-N2 & $86.7(4)$ & $86.1(2)$ & $85.6(2)$ & $82.05(13)$ & $82.74(14)$ \\
\hline N1-Mn1-N3 & $169.8(5)$ & $168.7(3)$ & $167.6(2)$ & $159.22(12)$ & $160.58(13)$ \\
\hline N2-Mn1-N3 & $85.1(4)$ & $84.2(3)$ & $83.7(2)$ & $79.21(14)$ & $79.43(16)$ \\
\hline N4-Mn1-N1 & $98.8(5)$ & $100.2(3)$ & $102.8(2)$ & 113.27(12) & $111.50(12)$ \\
\hline
\end{tabular}




\begin{tabular}{|c|c|c|c|c|c|}
\hline N4-Mn1-N2 & $173.5(5)$ & $173.0(2)$ & $171.0(2)$ & $164.39(13)$ & $165.45(13)$ \\
\hline N4-Mn1-N3 & $89.8(5)$ & $89.8(3)$ & $88.2(2)$ & $86.08(12)$ & $86.81(14)$ \\
\hline O3-Mn2-O4 & $174.8(5)$ & $175.8(2)$ & $176.3(2)$ & $176.32(11)$ & \\
\hline O3-Mn2-N5 & $90.8(5)$ & $90.7(3)$ & $89.5(2)$ & $88.22(11)$ & \\
\hline O3-Mn2-N6 & $83.8(5)$ & $84.5(2)$ & $94.4(2)$ & $94.04(11)$ & \\
\hline O3-Mn2-N7 & $92.6(5)$ & $92.8(3)$ & $84.6(2)$ & $85.60(11)$ & \\
\hline O3-Mn2-N8 & $90.6(5)$ & $89.8(2)$ & $90.4(2)$ & $89.76(11)$ & \\
\hline O4-Mn2-N5 & $88.6(5)$ & $88.5(3)$ & $94.0(2)$ & $93.11(11)$ & \\
\hline O4-Mn2-N6 & $91.1(5)$ & $91.3(2)$ & $87.2(2)$ & $89.53(11)$ & \\
\hline O4-Mn2-N7 & $87.4(4)$ & $87.5(2)$ & $92.2(2)$ & $94.19(11)$ & \\
\hline O4-Mn2-N8 & $94.6(5)$ & $94.4(2)$ & $87.5(2)$ & $86.56(11)$ & \\
\hline N5-Mn2-N6 & $89.3(5)$ & $88.9(3)$ & $84.0(2)$ & $82.83(11)$ & \\
\hline N5-Mn2-N7 & $172.6(5)$ & $172.7(3)$ & $164.9(2)$ & $160.85(11)$ & \\
\hline N7-Mn2-N6 & $100.1(5)$ & $100.4(3)$ & $82.5(2)$ & $79.55(11)$ & \\
\hline N8-Mn2-N5 & $84.6(5)$ & $85.0(3)$ & $105.9(2)$ & $111.04(11)$ & \\
\hline N8-Mn2-N6 & $169.1(5)$ & $169.2(3)$ & $169.0(2)$ & $165.75(11)$ & \\
\hline N8-Mn2-N7 & $86.4(5)$ & $86.1(3)$ & $88.1(2)$ & $87.07(11)$ & \\
\hline O5-Mn3-O6 & & & $176.1(2)$ & & \\
\hline O5-Mn3-N9 & & & $88.0(2)$ & & \\
\hline O5-Mn3-N10 & & & $94.35(19)$ & & \\
\hline O5-Mn3-N11 & & & $85.09(19)$ & & \\
\hline O5-Mn3-N12 & & & $89.8(2)$ & & \\
\hline O6-Mn3-N9 & & & $92.79(19)$ & & \\
\hline O6-Mn3-N10 & & & $89.57(19)$ & & \\
\hline O6-Mn3-N11 & & & $95.4(2)$ & & \\
\hline O6-Mn3-N12 & & & $86.3(2)$ & & \\
\hline N9-Mn3-N10 & & & $82.3(2)$ & & \\
\hline N9-Mn3-N11 & & & $159.5(2)$ & & \\
\hline N11-Mn3-N10 & & & $79.0(2)$ & & \\
\hline N12-Mn3-N9 & & & $111.9(2)$ & & \\
\hline N12-Mn3-N10 & & & $165.3(2)$ & & \\
\hline N12-Mn3-N11 & & & $87.3(2)$ & & \\
\hline O7-Mn4-08 & & & $176.8(2)$ & & \\
\hline O7-Mn4-N13 & & & $87.8(2)$ & & \\
\hline O7-Mn4-N14 & & & $95.0(2)$ & & \\
\hline O7-Mn4-N15 & & & $84.9(2)$ & & \\
\hline O7-Mn4-N16 & & & $90.6(2)$ & & \\
\hline O8-Mn4-N13 & & & $93.3(2)$ & & \\
\hline O8-Mn4-N14 & & & $88.2(2)$ & & \\
\hline O8-Mn4-N15 & & & $95.1(2)$ & & \\
\hline O8-Mn4-N16 & & & $86.2(2)$ & & \\
\hline N13-Mn4-N14 & & & $81.9(3)$ & & \\
\hline
\end{tabular}


N13-Mn4-N15

N15-Mn4-N14

N16-Mn4-N13

N16-Mn4-N14

N16-Mn4-N15
158.6(2)

78.7(3)

114.3(2)

163.1(2)

$86.0(2)$ 


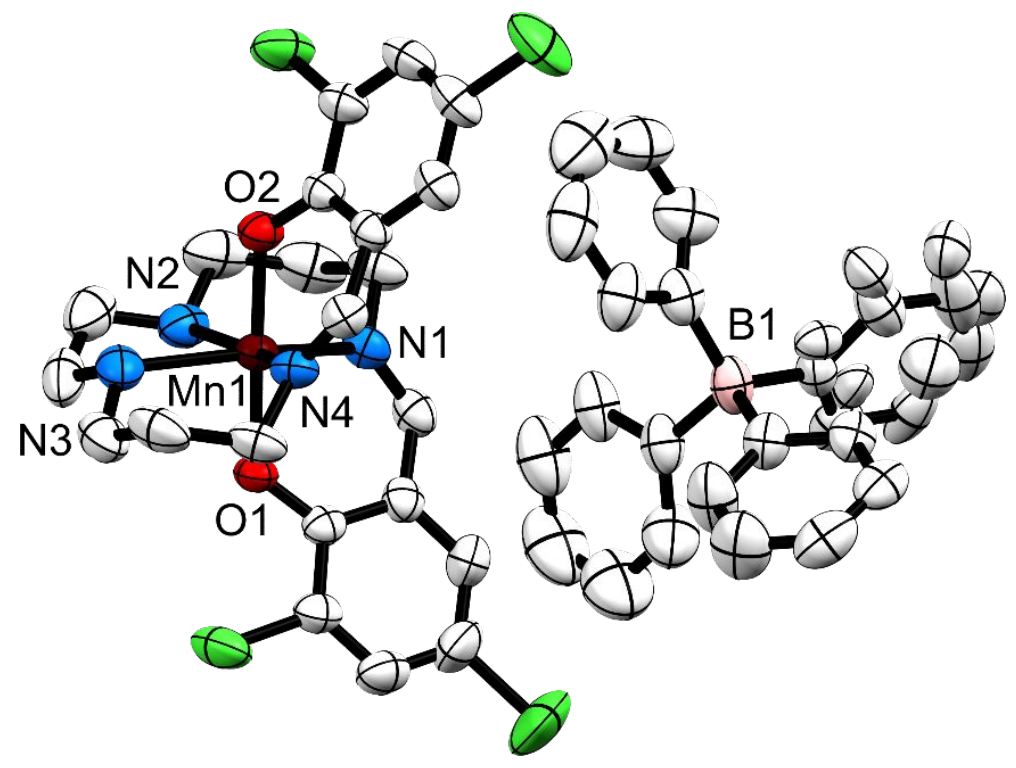

Figure S3 Asymmetric unit of complex 1, [Mn(3,5-Cl-sal $2(323))] \mathrm{BPh}_{4}$ measured at $250 \mathrm{~K}$ shown with $50 \%$ atomic probability distributions for ellipsoids with hydrogen atoms omitted for clarity.
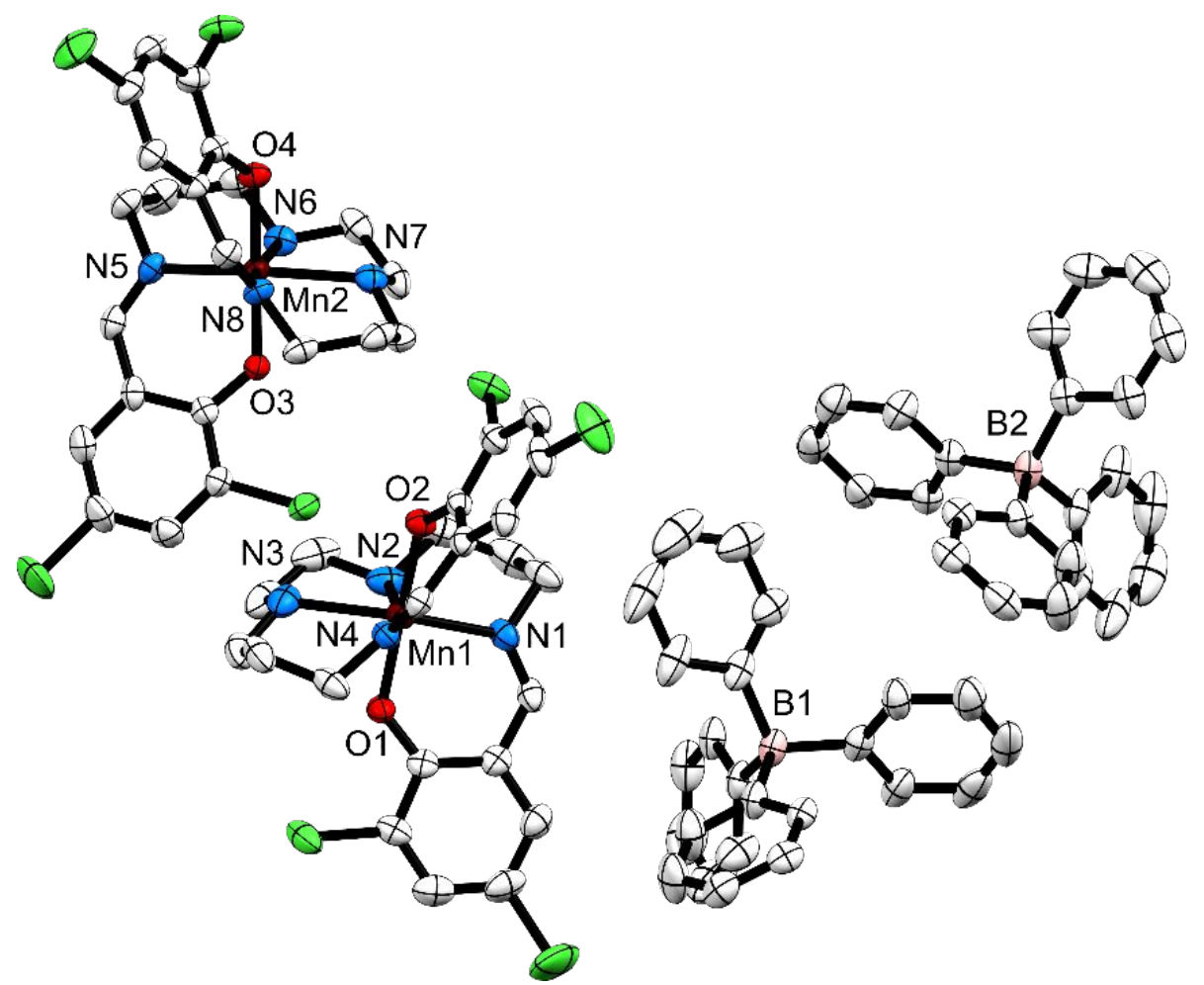

Figure S4 Asymmetric unit of complex 1, [Mn(3,5-Cl-sal $2(323))] \mathrm{BPh}_{4}$ measured at $150 \mathrm{~K}$ shown with $50 \%$ atomic probability distributions for ellipsoids with hydrogen atoms omitted for clarity. 


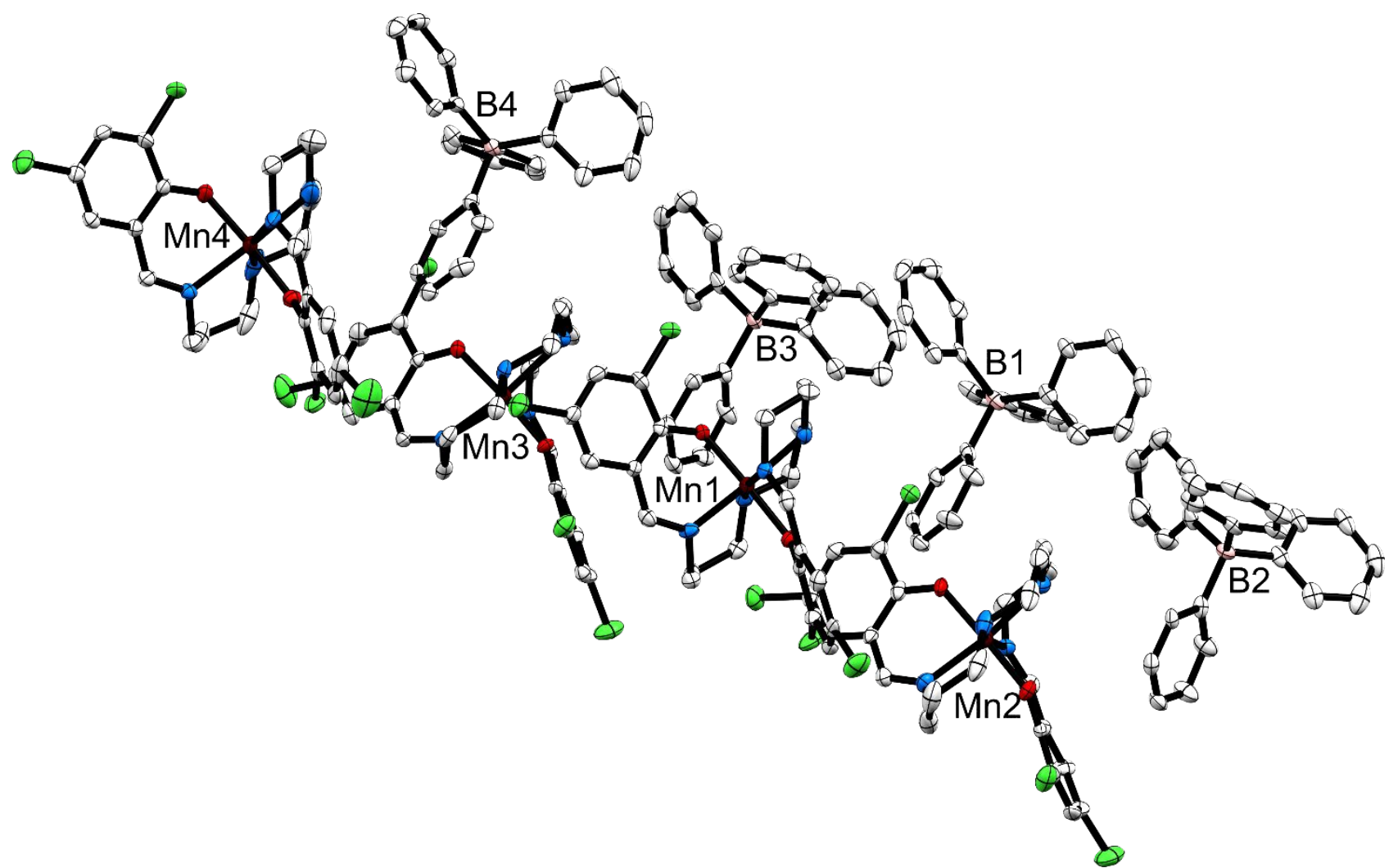

Figure S5 Asymmetric unit of complex 1, [Mn(3,5-diCl-sal) $\left.{ }_{2} 323\right] \mathrm{BPh}_{4}$ measured at $115 \mathrm{~K}$ shown with $50 \%$ atomic probability distributions for ellipsoids with hydrogen atoms omitted for clarity.

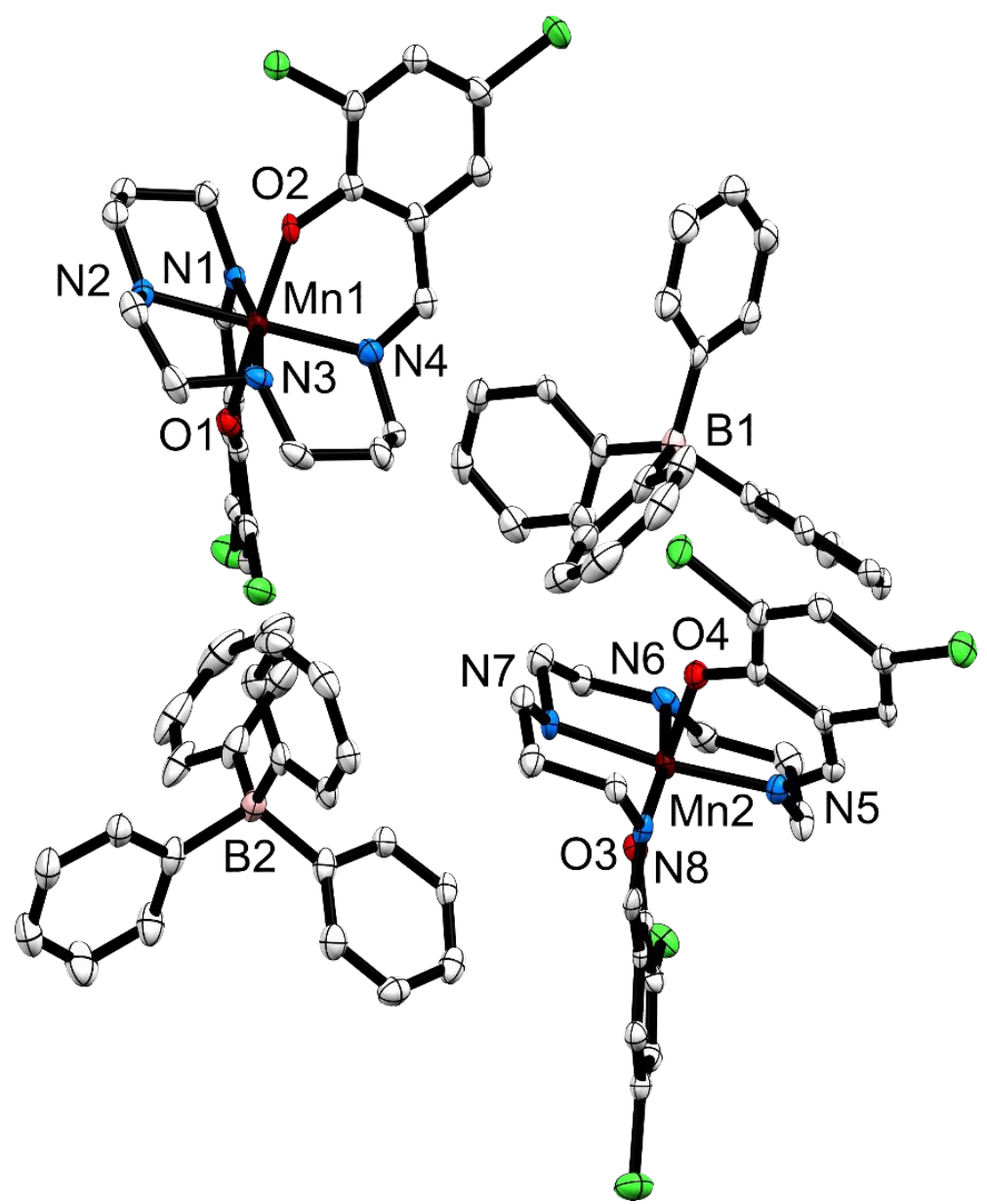

Figure S6 Asymmetric unit of complex 1, $\left[\mathrm{Mn}\left(3,5-\mathrm{Cl}_{-} \mathrm{sal}_{2}(323)\right)\right] \mathrm{BPh}_{4}$ measured at $82 \mathrm{~K}$ shown with $50 \%$ atomic probability distributions for ellipsoids with hydrogen atoms omitted for clarity. 

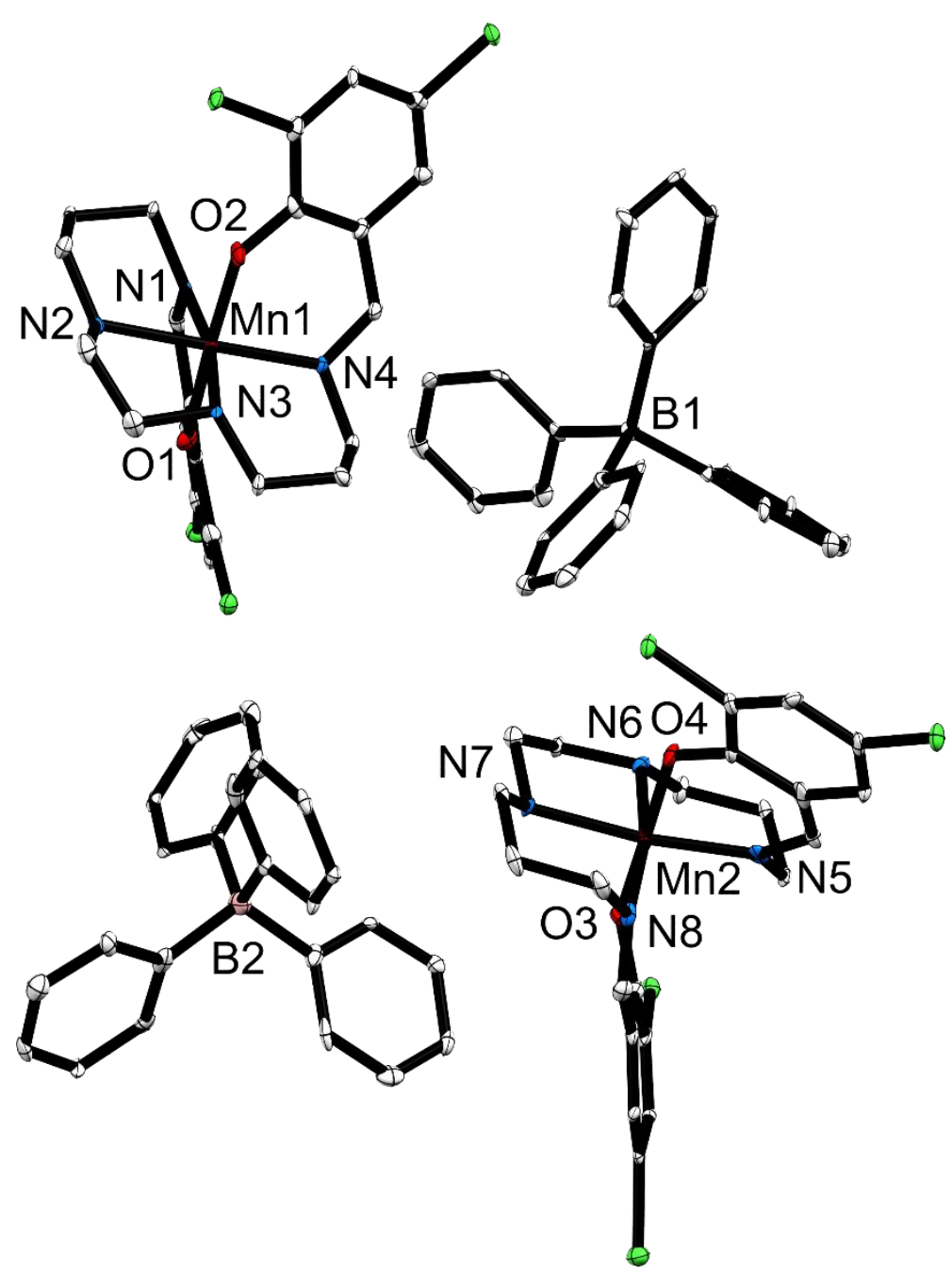

Figure S7 Asymmetric unit of complex 1, [ $\left.\mathrm{Mn}\left(3,5-\mathrm{Cl}_{-} \mathrm{sal}_{2}(323)\right)\right] \mathrm{BPh}_{4}$ measured at $10 \mathrm{~K}$ shown with $50 \%$ atomic probability distributions for ellipsoids with hydrogen atoms omitted for clarity.

Complex $\mathbf{1}$ is refined in the non-centrosymmetric polar space group $C_{c}$ at $250 \mathrm{~K}$. At $150 \mathrm{~K}$, complex $\mathbf{1}$ is refined in the non-centrosymmetric polar space group $P c$ and at $115 \mathrm{~K}, 82 \mathrm{~K}$ and $10 \mathrm{~K}$ in the noncentrosymmetric chiral and polar space group $P 1$. Complex 1 comprises the $\left[\mathrm{Mn}\left(3,5-\mathrm{diCl}_{-} \mathrm{sal}_{2}(323)\right)\right]^{+}$cation and the tetraphenylborate counteranion, $\mathrm{BPh}_{4}{ }^{-}$. Each unit cell in the HT, INT2 and INT1 phases contains four $\mathrm{Mn}^{3+}$ cations and four $\mathrm{BPh}_{4}{ }^{-}$anions. Hence, the $C c$ structures have $\mathrm{Z}=4$ and $\mathrm{Z}^{\prime}=1$, the $P c$ structure has $\mathrm{Z}=4$ and $Z^{\prime}=2$ and the $P 1$ structure has $Z=4$ and $Z^{\prime}=4$. The $L T P 1$ structure contains two $\mathrm{Mn}^{3+}$ cations and two $\mathrm{BPh}_{4}{ }^{-}$anions in the unit cell which results in $Z=2$ and $Z^{\prime}=2$. The structures are all polar (with the $P 1$ structure also being chiral) with Flack parameters ${ }^{[9]}$ of each structure being close to zero. No solvent molecules were located in any of the structures. 
The Hirshfeld surfaces are mapped with $d_{n o r m}$, and 2D fingerprint plots were generated using CrystalExplorer 17.5. ${ }^{1}$ The graphical plot uses a red-white-blue colour scheme for the molecular Hirshfeld surfaces. The red highlights on the Hirshfeld surfaces show contacts shorter than the van der Waals distance, contacts within the van der Waals distance are shown in white and longer contacts are shown in blue.

a) Hirshfeld surface mapped with $d_{\text {norm }}$ viewed along:
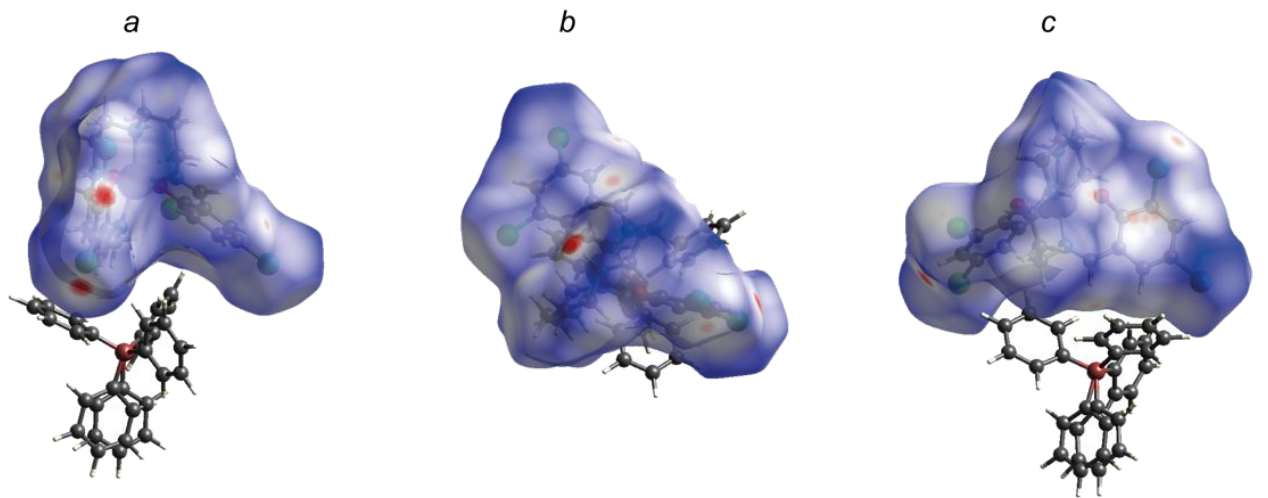

b) Fingerprint plots of contacts of $\mathbf{1}$ :
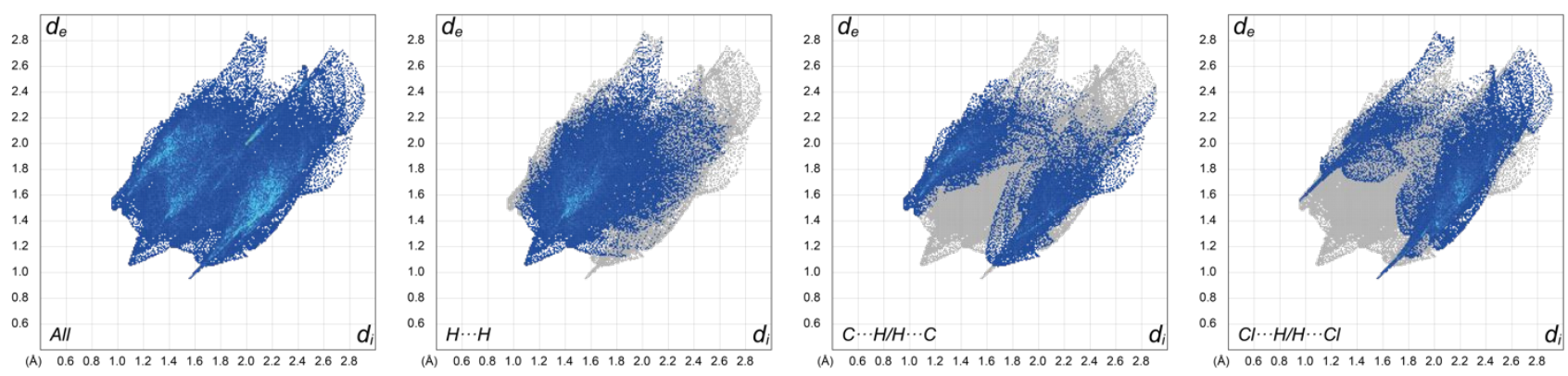

Figure S8 a) Hirshfeld surface mapped with $d_{\text {norm }}$ for the structure of complex 1 measured at $250 \mathrm{~K}$ viewed along the $a, b$ and $c$ direction, b) fingerprint plots with all intermolecular interactions resolved into the contribution of $\mathrm{H} \cdots \mathrm{Cl} / \mathrm{Cl} \cdots \mathrm{H}, \mathrm{H} \cdots \mathrm{C} / \mathrm{C} \cdots \mathrm{H}$ and $\mathrm{H} \cdots \mathrm{H} / \mathrm{H} \cdots \mathrm{H}$ contacts of complex 1 at $250 \mathrm{~K}$. 
a) Hirshfeld surface mapped with $d_{\text {norm }}$ viewed along:
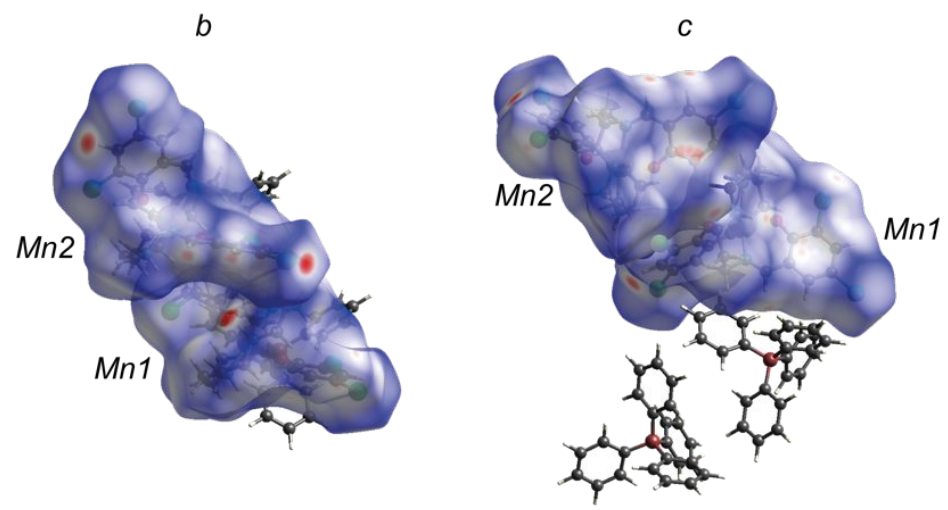

b) Fingerprint plots of contacts of $\mathbf{1}$ :

Mn1

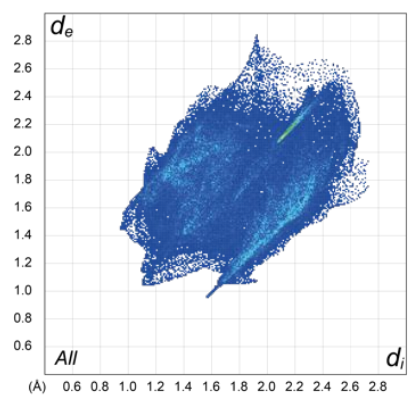

Mn2

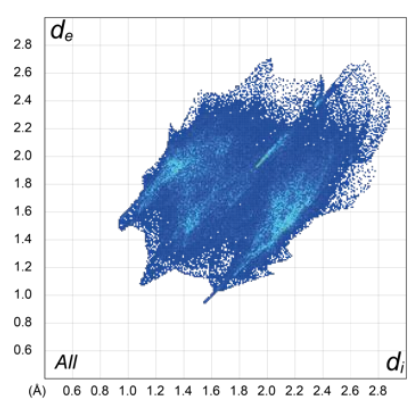

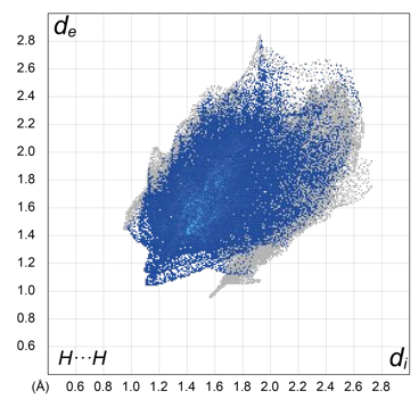
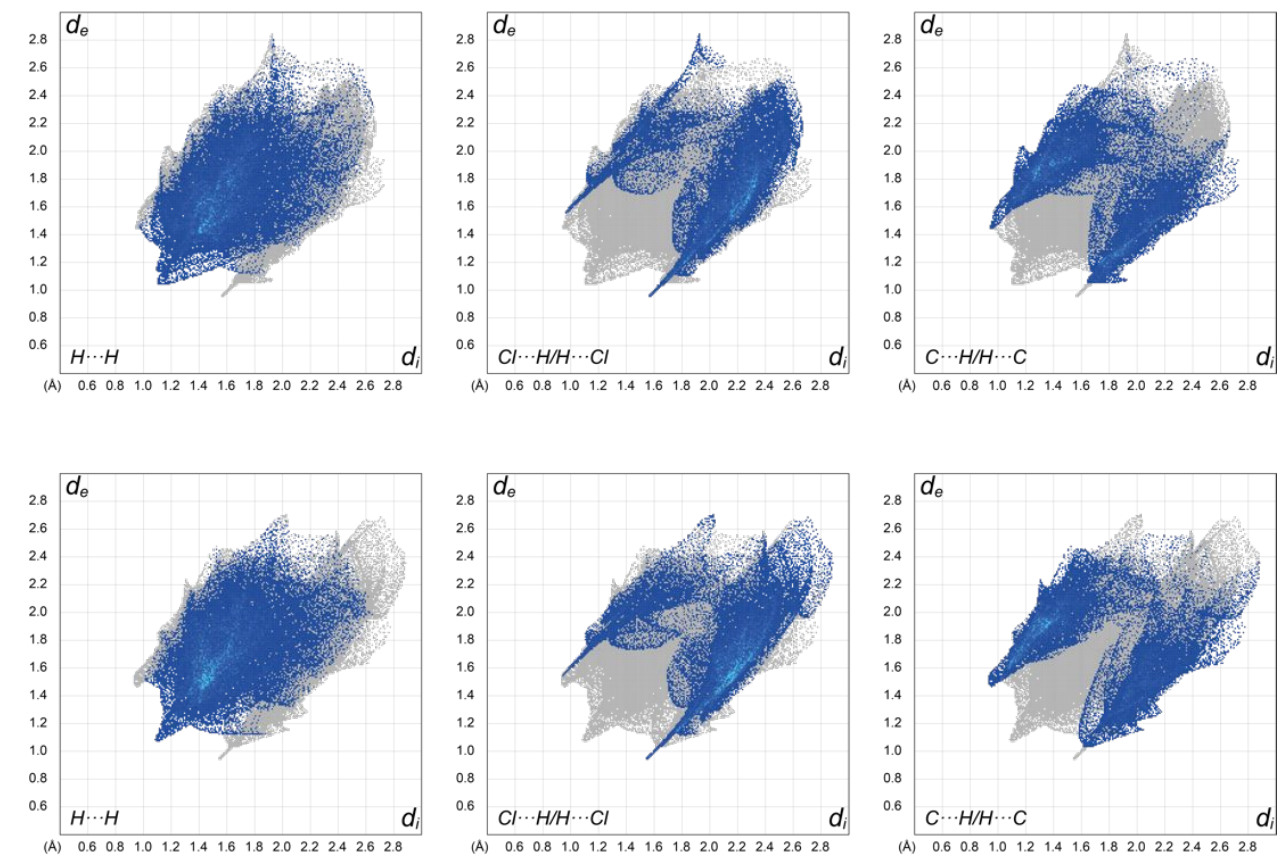
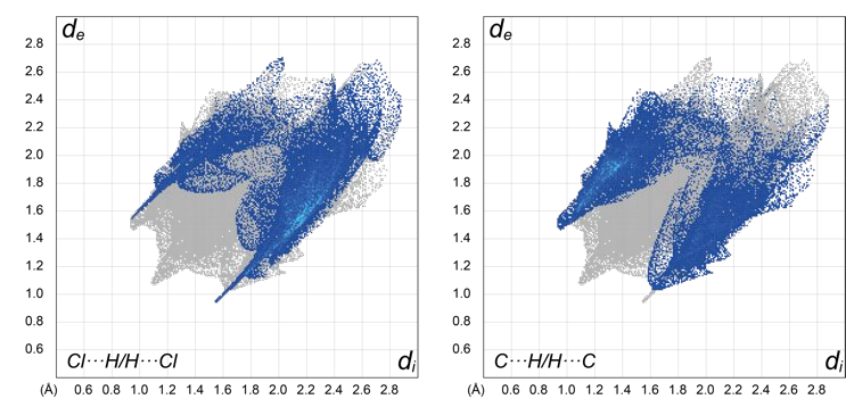

Figure S9 a) Hirshfeld surface mapped with $d_{\text {norm }}$ for the structure of complex 1 measured at $150 \mathrm{~K}$ viewed along the $a, b$ and $c$ direction, b) fingerprint plots with all intermolecular interactions resolved into the contribution of $\mathrm{H} \cdots \mathrm{Cl} / \mathrm{Cl} \cdots \mathrm{H}, \mathrm{H} \cdots \mathrm{C} / \mathrm{C} \cdots \mathrm{H}$ and $\mathrm{H} \cdots \mathrm{H} / \mathrm{H} \cdots \mathrm{H}$ contacts of complex 1 at $150 \mathrm{~K}$. 
a) Hirshfeld surface mapped with $d_{\text {norm }}$ viewed along:

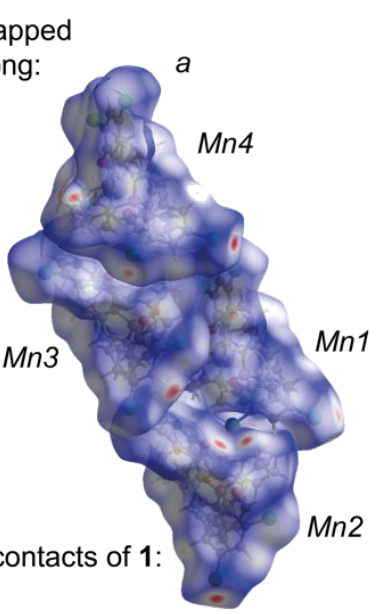

Mn1
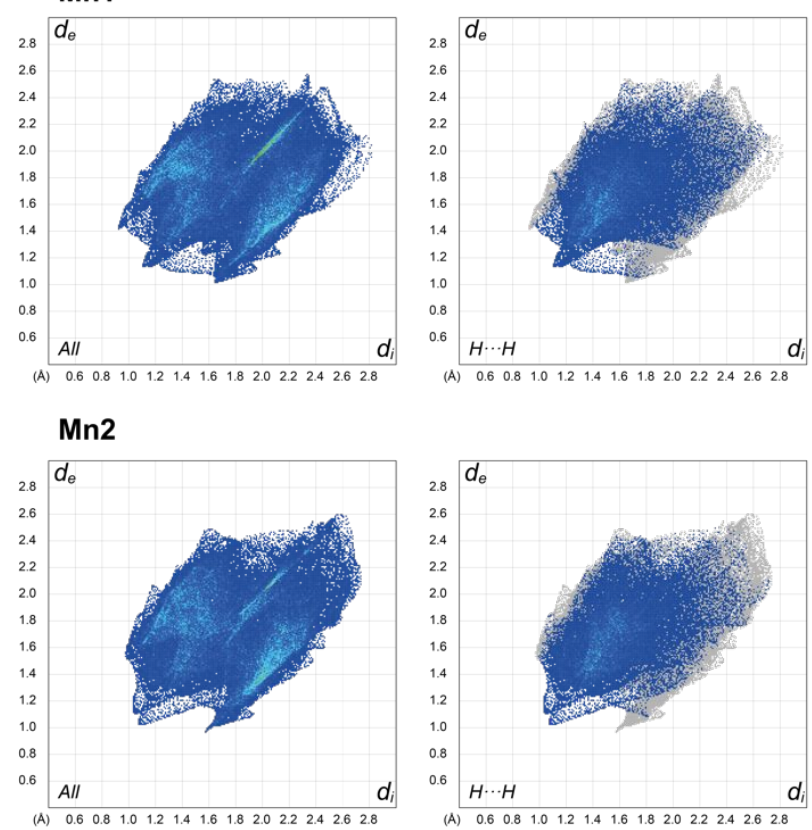

Mn3

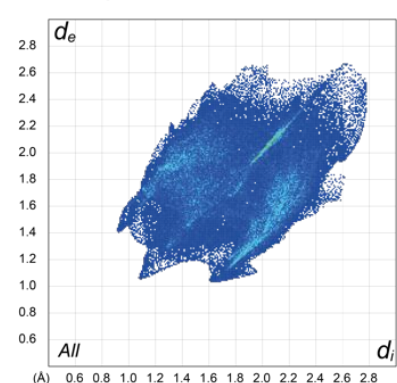

Mn4
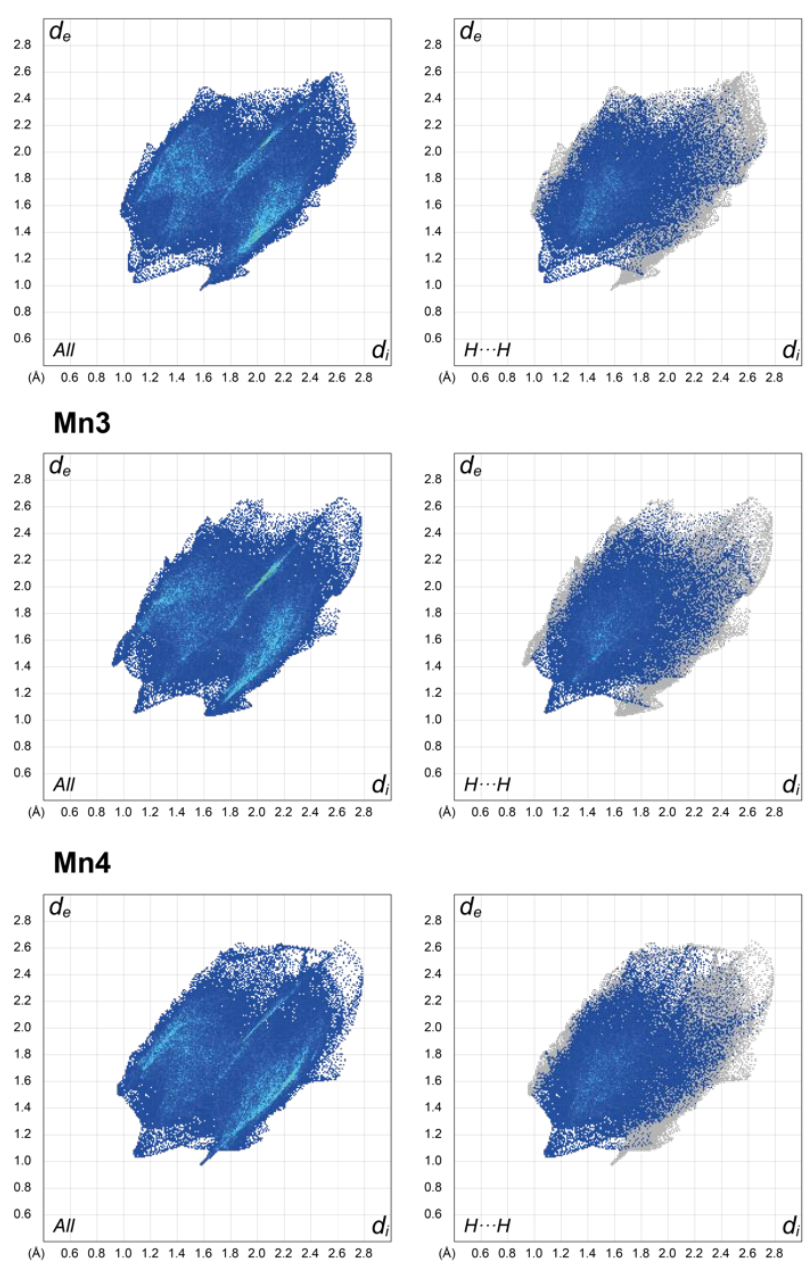
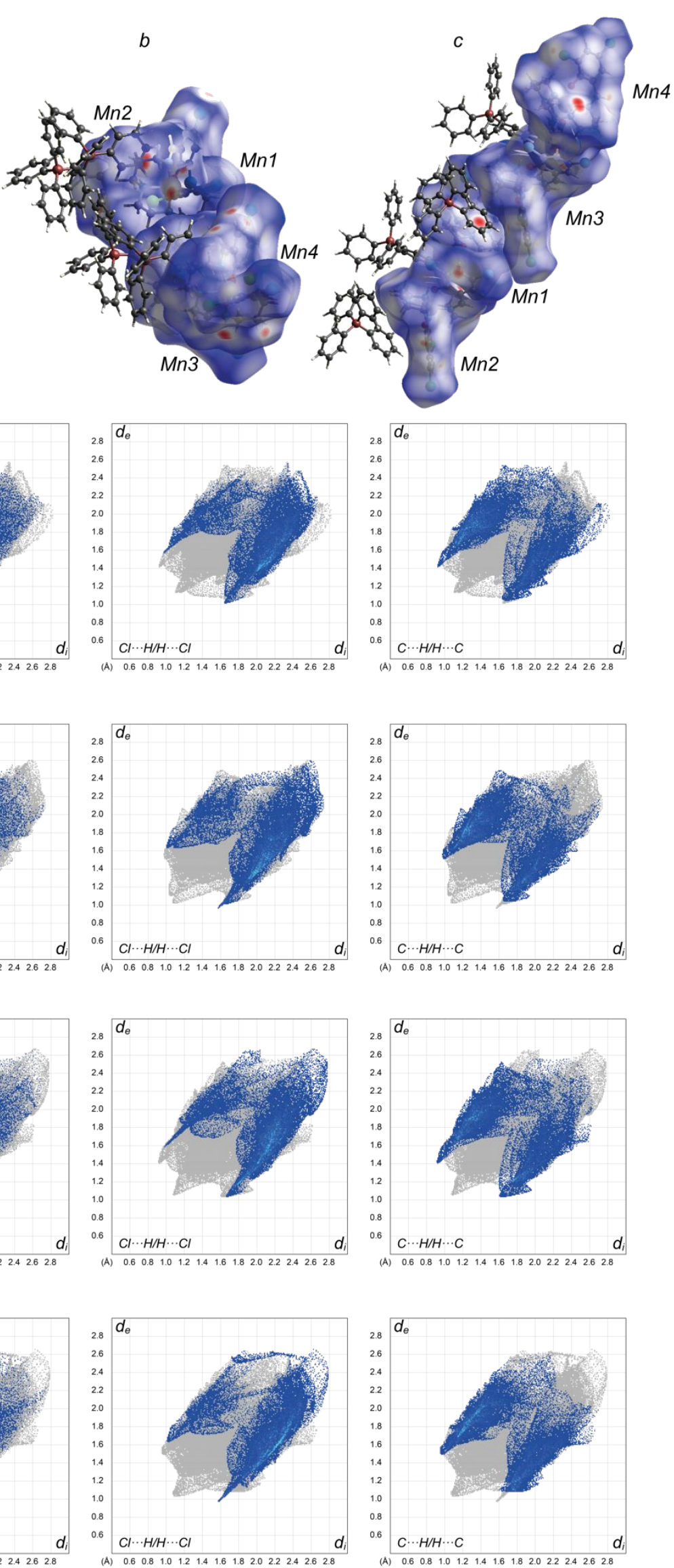

Figure S10 a) Hirshfeld surface mapped with $d_{\text {norm }}$ for the structure of complex 1 measured at $115 \mathrm{~K}$ viewed along the $a, b$ and $c$ direction, b) fingerprint plots with all intermolecular interactions resolved into the contribution of $\mathrm{H} \cdots \mathrm{Cl} / \mathrm{Cl} \cdots \mathrm{H}, \mathrm{H} \cdots \mathrm{C} / \mathrm{C} \cdots \mathrm{H}$ and $\mathrm{H} \cdots \mathrm{H} / \mathrm{H} \cdots \mathrm{H}$ contacts of complex 1 at $115 \mathrm{~K}$. 
a) Hirshfeld surface mapped with $d_{\text {norm }}$ viewed along:

b) Fingerprint plots of contacts of 1 :
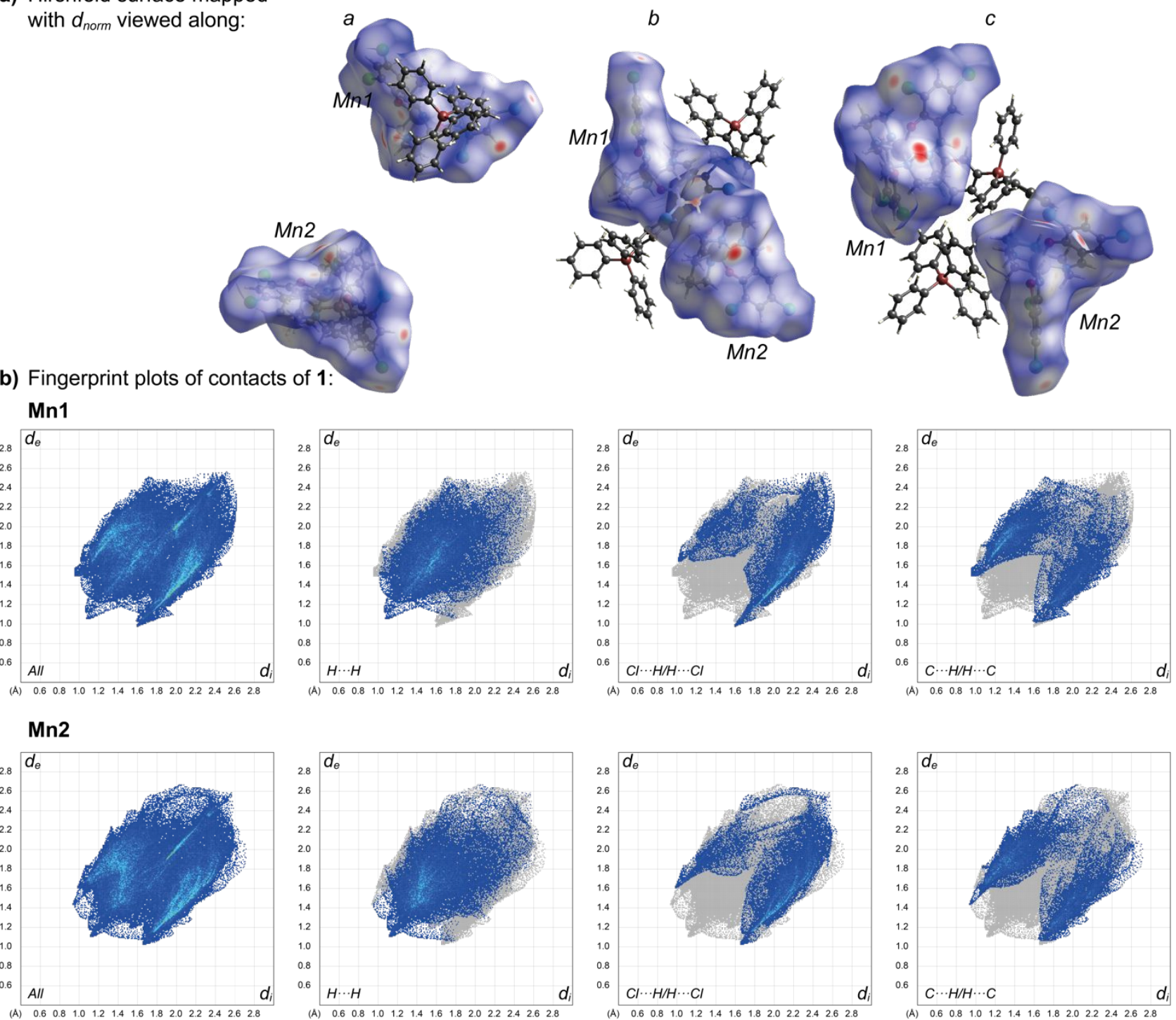

Figure S11 a) Hirshfeld surface mapped with $d_{\text {norm }}$ for the structure of complex 1 measured at $82 \mathrm{~K}$ viewed along the $a, b$ and $c$ direction, b) fingerprint plots with all intermolecular interactions resolved into the contribution of $\mathrm{H} \cdots \mathrm{Cl} / \mathrm{Cl} \cdots \mathrm{H}, \mathrm{H} \cdots \mathrm{C} / \mathrm{C} \cdots \mathrm{H}$ and $\mathrm{H} \cdots \mathrm{H} / \mathrm{H} \cdots \mathrm{H}$ contacts of complex 1 at $82 \mathrm{~K}$. 
a) Hirshfeld surface mapped with $d_{\text {norm }}$ viewed along:

b) Fingerprint plots of contacts of 1 :

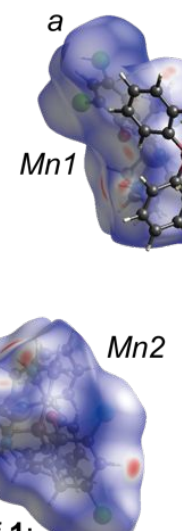

Mn1

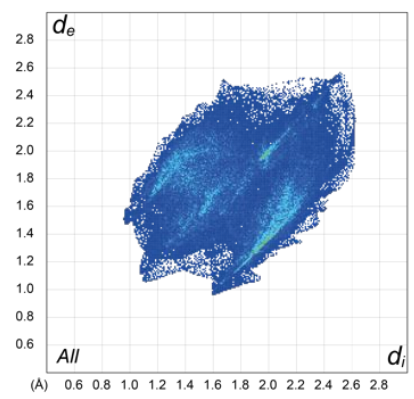

Mn2

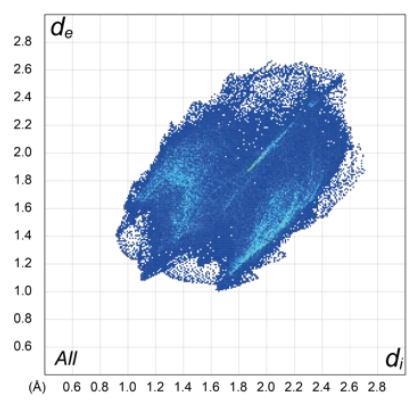

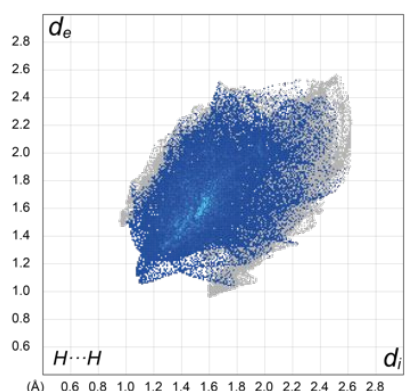
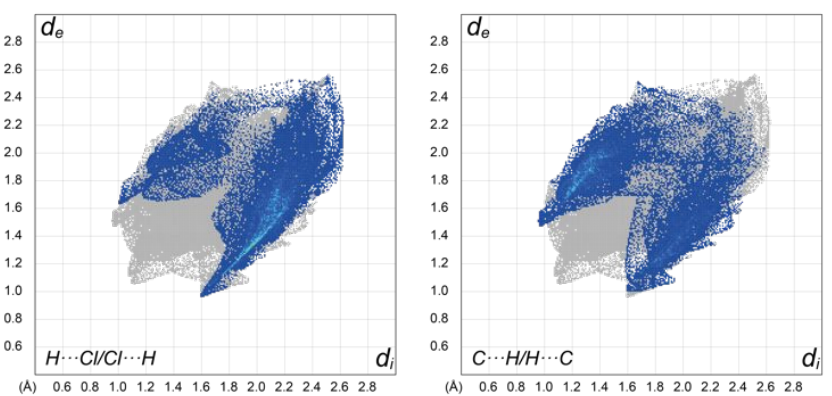
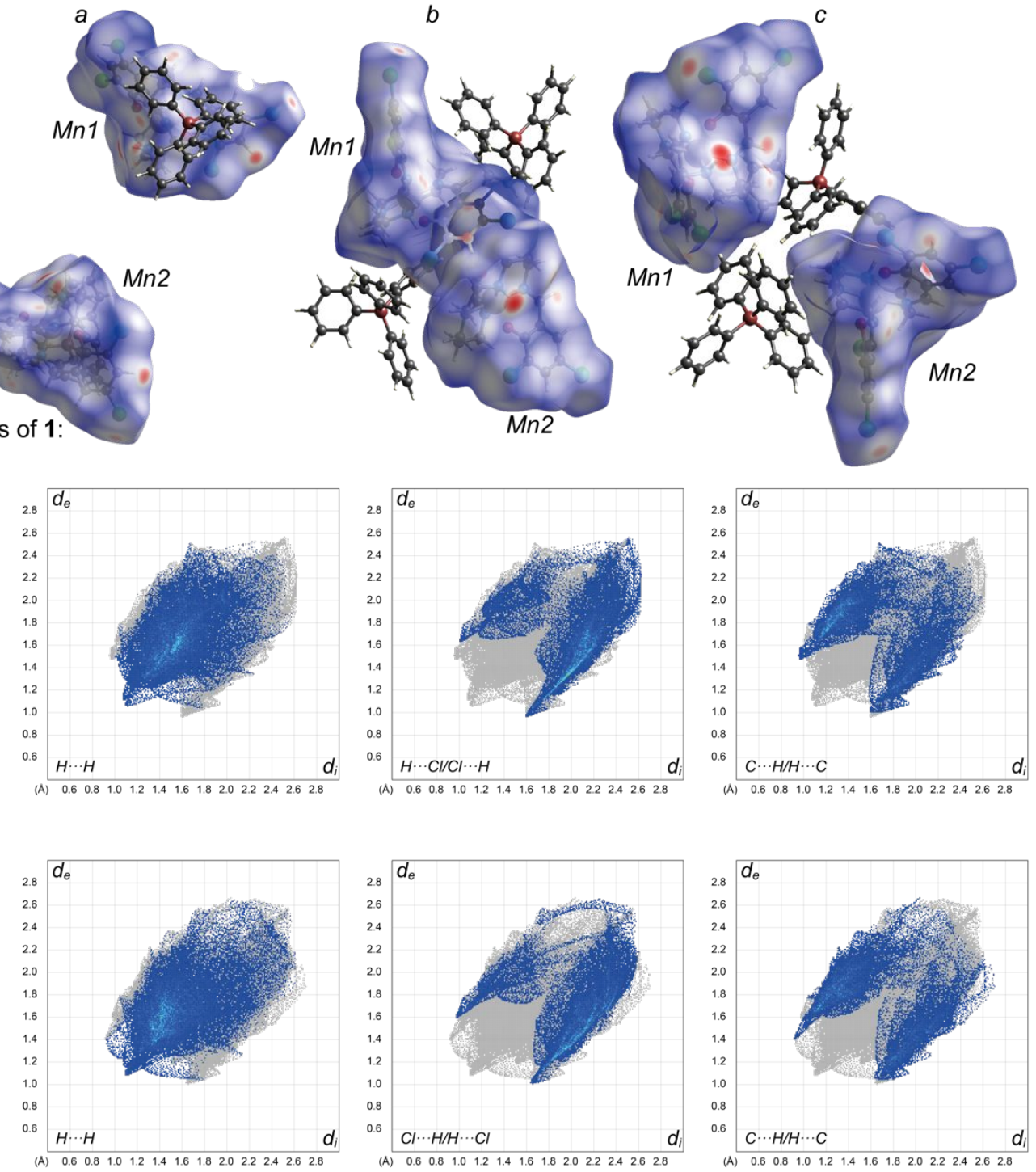

Figure S12 a) Hirshfeld surface mapped with $d_{\text {norm }}$ for the structure of complex 1 measured at $10 \mathrm{~K}$ viewed along the $a, b$ and $c$ direction, b) fingerprint plots with all intermolecular interactions resolved into the contribution of $\mathrm{H} \cdots \mathrm{Cl} / \mathrm{Cl} \cdots \mathrm{H}, \mathrm{H} \cdots \mathrm{C} / \mathrm{C} \cdots \mathrm{H}$ and $\mathrm{H} \cdots \mathrm{H} / \mathrm{H} \cdots \mathrm{H}$ contacts of complex 1 at $10 \mathrm{~K}$. 


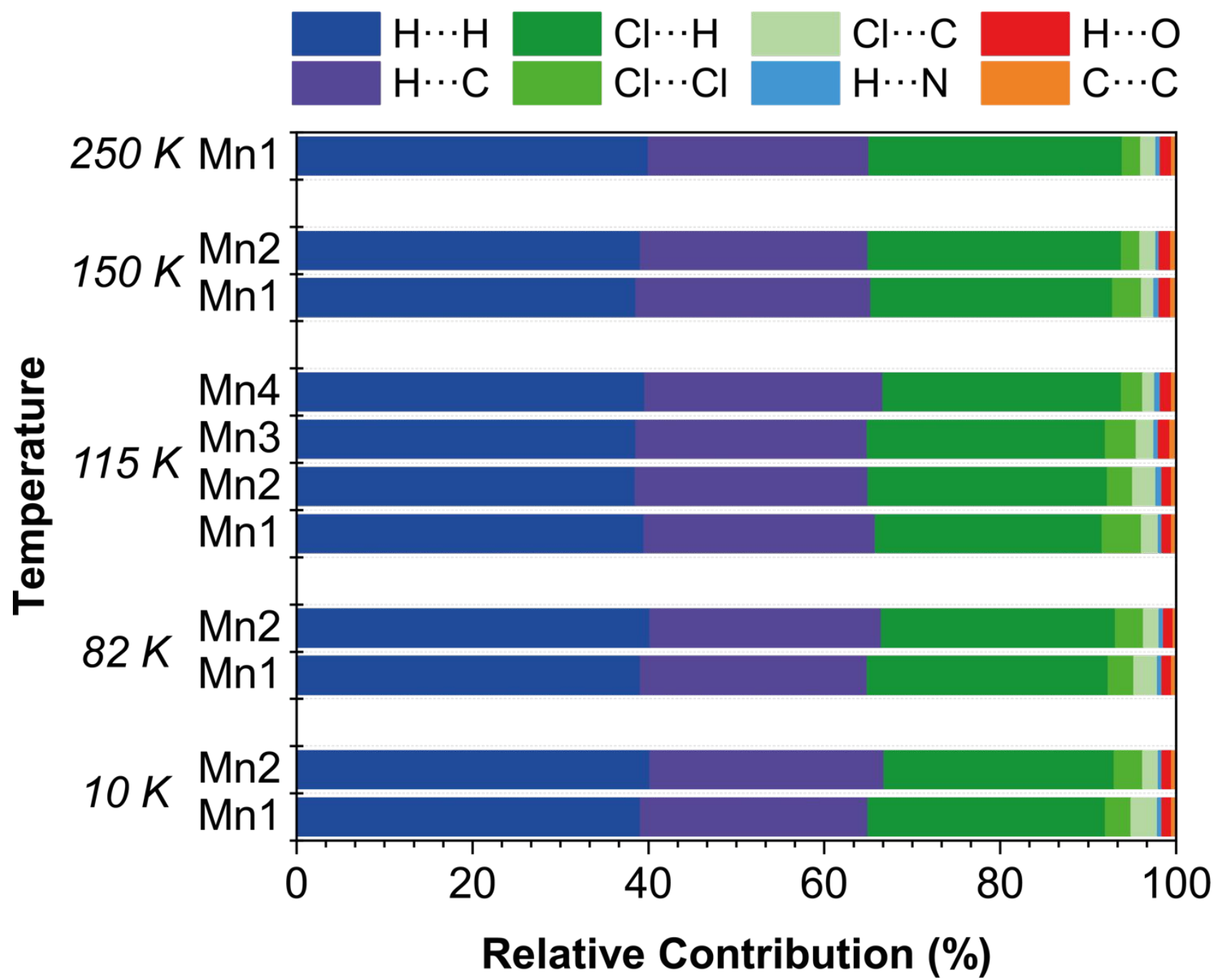

Figure S13 Relative contributions to the Hirshfeld surface area of different intermolecular interactions for the structures at $10,82,115,150$ and $250 \mathrm{~K}$.

The dominating interactions originate from $\mathrm{H} \cdots \mathrm{C}, \mathrm{H} \cdots \mathrm{Cl}$, and $\mathrm{H} \cdots \mathrm{H}$ between the hydrogen atoms on the tetraphenylborate anion and the chloride groups on the phenyl ring on the salicylaldehyde motif as well as the hydrogen and carbon atoms in the backbone of the $\mathrm{Mn}^{3+}$ chelated complex 1 . Those interactions appear as distinct spikes in the 2D fingerprint plot. 
S2.4 Variable temperature single crystal X-ray diffraction
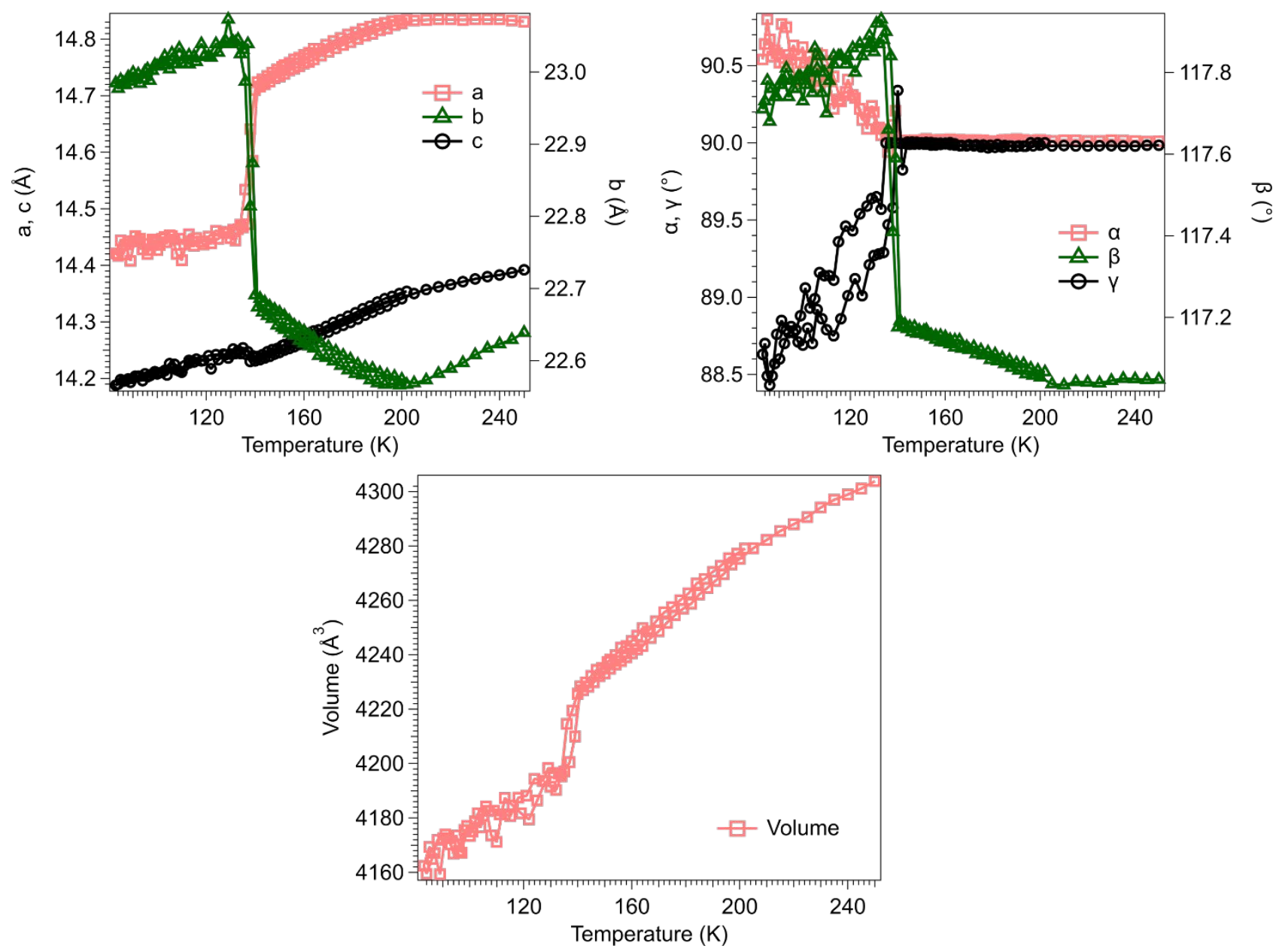

Figure S14 Variable temperature X-ray diffraction of changes in unit cell parameters, $V, a, b, c, \alpha, b$ and $\gamma$, measured on a single crystal of complex 1 showing the cooling and warming dependence between $250 \mathrm{~K} \rightarrow$ $83 \mathrm{~K} \rightarrow 200 \mathrm{~K}$. The data was integrated with respect to the HT Cc cell at $250 \mathrm{~K}$. 


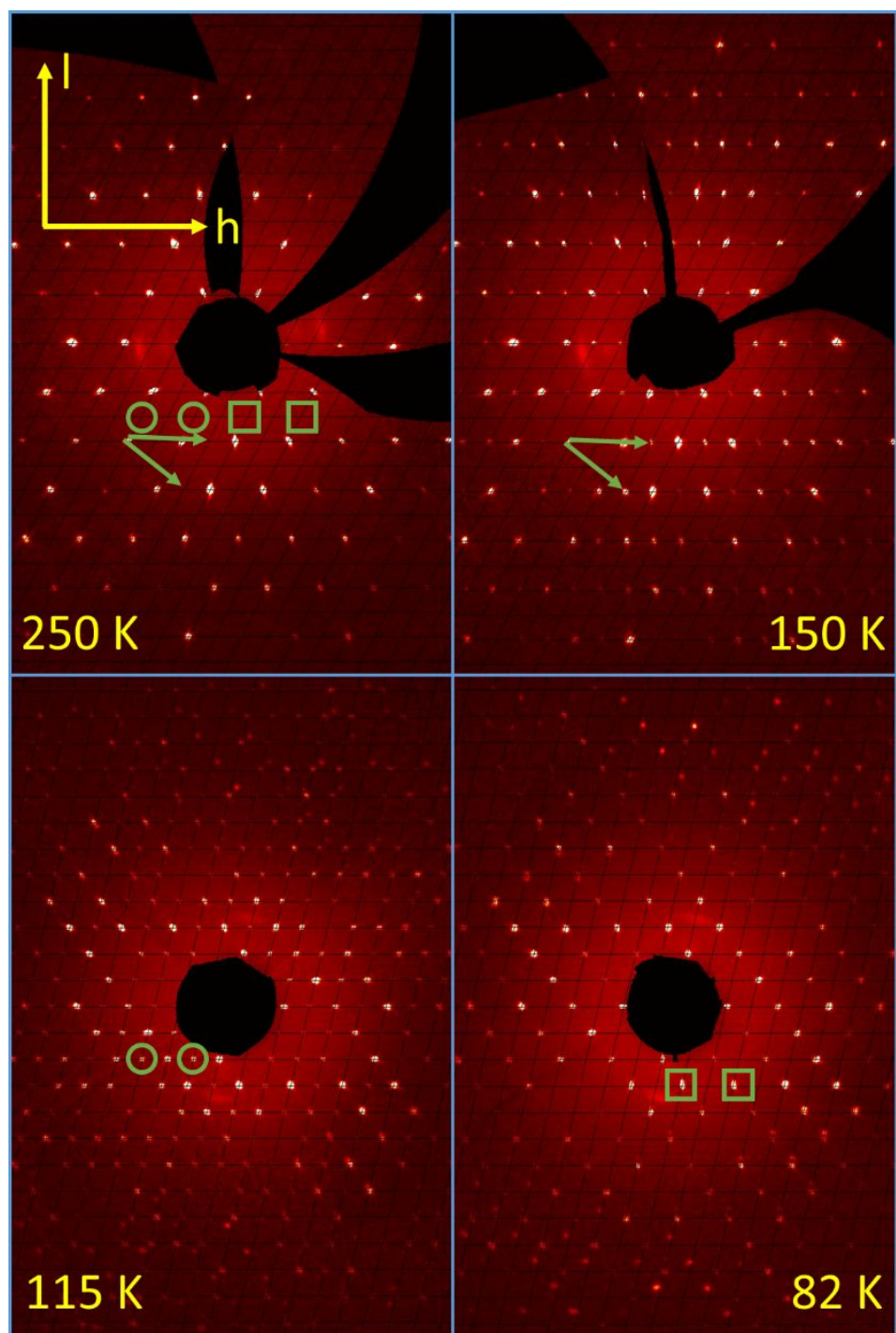

Figure S15 Representative precession images (h0l) plane obtained from variable temperature single crystal $\mathrm{X}$-ray diffraction of the unit cell parameters collected on a single crystal of complex 1 at $250,150 \mathrm{~K}, 115 \mathrm{~K}$ and $82 \mathrm{~K}$. 


\section{S3.1 Strain analysis from unit cell parameters}

Spontaneous strains associated with the three structural phase transitions observed in complex $\mathbf{1}$ have been determined using the approach and equations as previously set out. ${ }^{2}$ The parent structure has space group $C c$ and the three derivative structures have space groups $P c, P 1$ and $P 1_{(1 / 2)}$ respectively. The groupsubgroup sequences are as follows: $C c \rightarrow P c$ (co-elastic), $C c \rightarrow P 1$ (improper ferroelastic) and $C c \rightarrow P 1_{(1 / 2)}$ (pseudoproper ferroelastic). Values of reference parameters $a_{0}, b_{0}, c_{0}, \alpha_{0}\left(=90^{\circ}\right), \beta_{0}{ }^{*}\left(=180-b_{0}\right), \gamma_{0}\left(=90^{\circ}\right)$ for the $C c$ structure were obtained by extrapolation of a linear fit to measured parameters in the temperature interval $250 \mathrm{~K}$ to $208 \mathrm{~K}$, Figure S16. A linear extrapolation does not allow for the requirement that the slope of lattice parameters of crystalline materials must tend to zero as $T \rightarrow 0$, but there is insufficient data for the CC structure to allow fitting with a coth function as would normally be used for analysis of strains at low temperatures. ${ }^{3-4}$ 

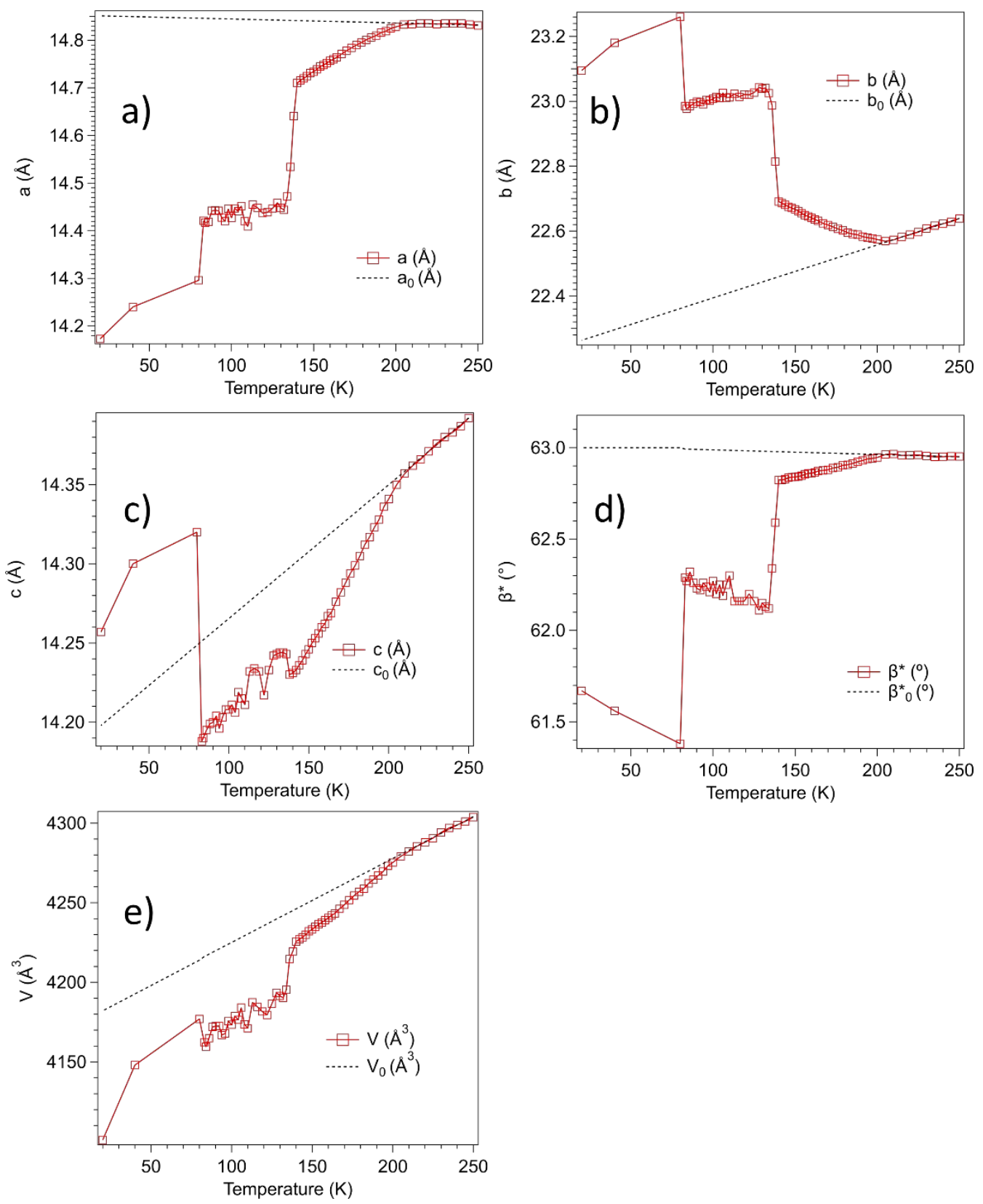

Figure S16 Linear fits to lattice parameter data in the temperature interval $250 \mathrm{~K}$ to $83 \mathrm{~K}$. The extrapolations to lower temperatures (dotted lines) represent variations of reference parameters $a_{0}, b_{0}, c_{0}, \alpha_{0}\left(=90^{\circ}\right), b_{0}, \gamma_{0}$ $\left(=90^{\circ}\right)$ in calculations of the spontaneous strains associated with $C c \rightarrow P c, C c \rightarrow P 1$ and $C c \rightarrow P 1_{(1 / 2)}$ transitions. Reference values of the unit cell volume, $V_{0}$, were obtained in the same way. 
Individual strain components, $e_{i}, i=1,2,3,5$ were calculated for $C c \rightarrow P c\left(e_{4}=e_{6}=0\right)$ according to the following equations:

$$
\begin{array}{r}
e_{1}=\frac{a-a_{0}}{a_{0}}(S 1) \\
e_{2}=\frac{b-b_{0}}{b_{0}}(S 2) \\
e_{3}=\frac{c \sin \beta-c_{0} \sin \beta_{0}}{c_{0} \sin \beta_{0}} \\
e_{5}=\left(\frac{c \cos \beta}{c_{0} \sin \beta_{0}}-\frac{a \cos \beta_{0}}{a_{0} \sin \beta_{0}}\right)
\end{array}
$$

Individual strain components, $e_{i}, i=1-6$ were calculated for $C c \rightarrow P 1$ and $C c \rightarrow P 1_{\left(\frac{1 / 2}{2}\right)}$ according to the following equations:

$$
\begin{gathered}
e_{1}=\frac{a}{a_{0}} \sin (\gamma)-1 \quad(S 5) \\
e_{2}=\frac{b}{b_{0}}-1 \quad(S 6) \\
e_{3}=\frac{c \sin (\alpha) \sin \left(\beta^{*}\right)}{c_{0} \sin \left(\beta_{0}^{*}\right)}-1 \quad(S 7) \\
e_{5}=\left(\frac{c \cos (\alpha)}{c_{0} \sin \left(\beta_{0}^{*}\right)}+\frac{a \cos \left(\beta_{0}^{*}\right) \cos (\gamma)}{a_{0} \sin \left(\beta_{0}^{*}\right)}\right) \\
e_{0} \sin (\gamma) \cos \left(\beta_{0}^{*}\right) \\
a_{0} \sin \left(\beta_{0}^{*}\right) \\
e_{6}=\left(\frac{c \sin (\alpha) \cos \left(\beta^{*}\right)}{c_{0} \sin \left(\beta_{0}^{*}\right)}\right) \\
\left.e_{0} \cos (\gamma)\right) \quad(S 10)
\end{gathered}
$$

Values of the volume strain, $V_{s}$, were given by the following equation:

$$
V_{s}=\frac{V-V_{0}}{V_{0}}
$$

The resulting strain variations are given in Figures $5 \mathrm{~g}, \mathrm{~h}$ of the main text. A test of the accuracy of linear extrapolations to obtain the reference parameters is provided by comparison of values for $V_{\mathrm{s}}$ obtained directly using Equation S11 with values obtained using $V_{s}=e_{1}+e_{2}+e_{3}$, which should be accurate for small volume strains. As shown in Figure $5 \mathrm{~g}$, the two variations of $V_{s}$ have the same non-linear form of temperature dependence but with different absolute values. It is safe to conclude that the strain components all have a 
continuous variation through the $C c \rightarrow P c$ transition point and a non-linear dependence on temperature in the stability field of the $P c$ structure. Errors in the absolute values must increase with falling temperature however, because of the assumption of linearity for the reference parameters. The $P c \rightarrow P 1$ transition is clearly discontinuous as expected, given that two space groups do not have a group-subgroup relationship. In principle it should be possible to determine the transition temperature for the $C c \rightarrow P 1$ transition by extrapolation of a fit to $e_{4}$ and $e_{6}$ to zero, but there is insufficient data to produce a reliable result.

For each of the $C c \rightarrow P c$ and $C c \rightarrow P 1$ transitions, the lowest order terms for coupling between strains, $e_{i}$, and the driving order parameter, $q$, have the form $\lambda e_{i} q^{2}$. This leads to the expected relationships $e_{i} \propto V_{s} \propto$ $q^{2}$. For the $C c \rightarrow P 1_{\left(\frac{1}{2}\right)}$ transition, the lowest order terms for coupling between strains, $e_{i}$, and the driving order parameter, $q$, have the form $\lambda e_{i} q$. This leads to the expected relationships $e_{\mathrm{i}} \propto V_{\mathrm{s}} \propto q$. $^{2}$ 


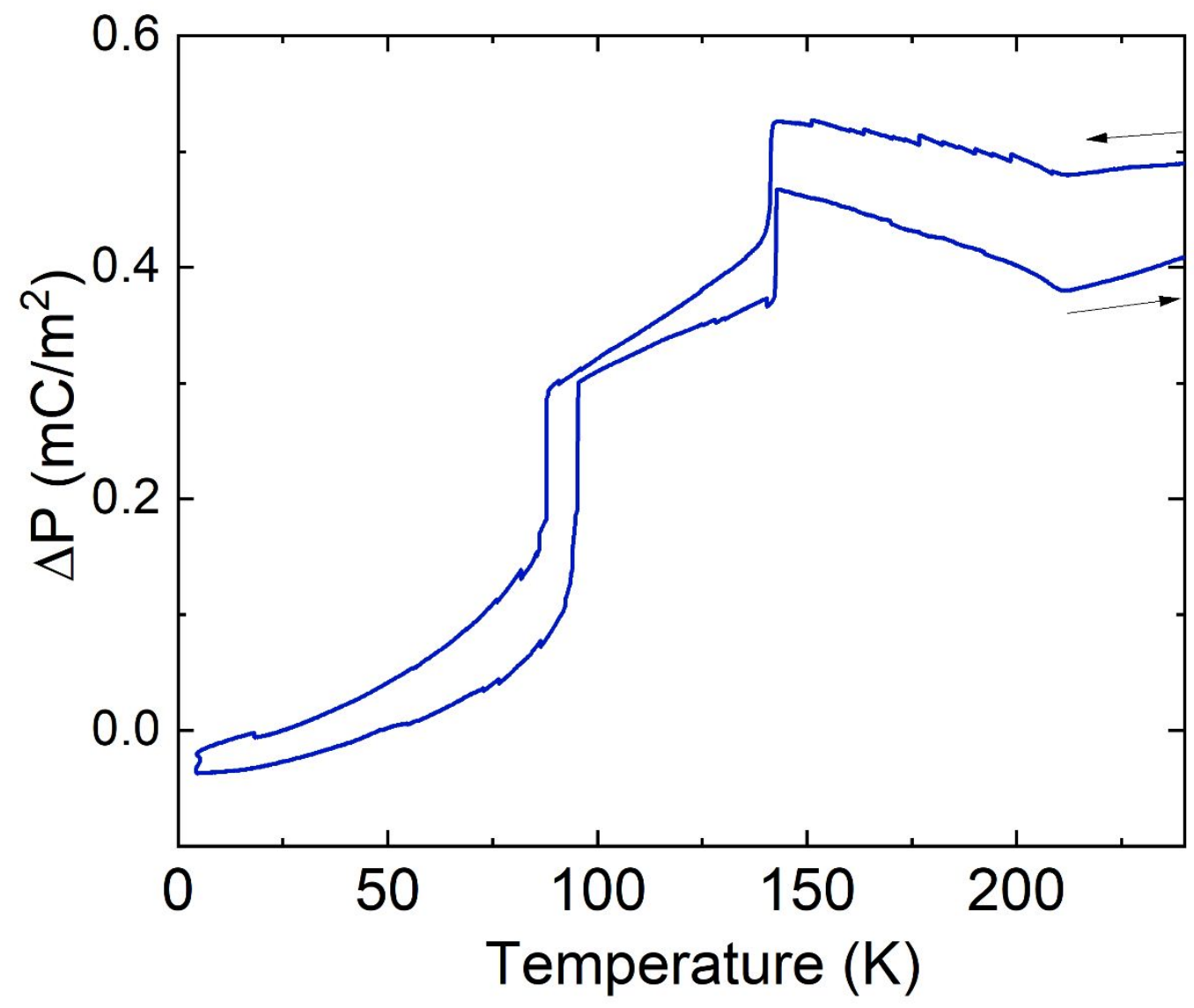

Figure S17 Electric polarization change $\Delta P$ measured vs temperature in zero externally applied electric and magnetic field for cooling and heating. The electric polarization shows sudden jumps at the LT $\rightarrow$ INT1 and INT1 $\rightarrow$ INT2 transitions, consistent with the change in polar space group. A kink occurs at the INT2 $\rightarrow$ HT transition. Small discontinuous jumps and irreversibility can be attributed to the interaction of the sample with the glue that adheres it to the measurement probe. 


\section{$\Delta P\left(\mathrm{mC} / \mathrm{m}^{2}\right)$}

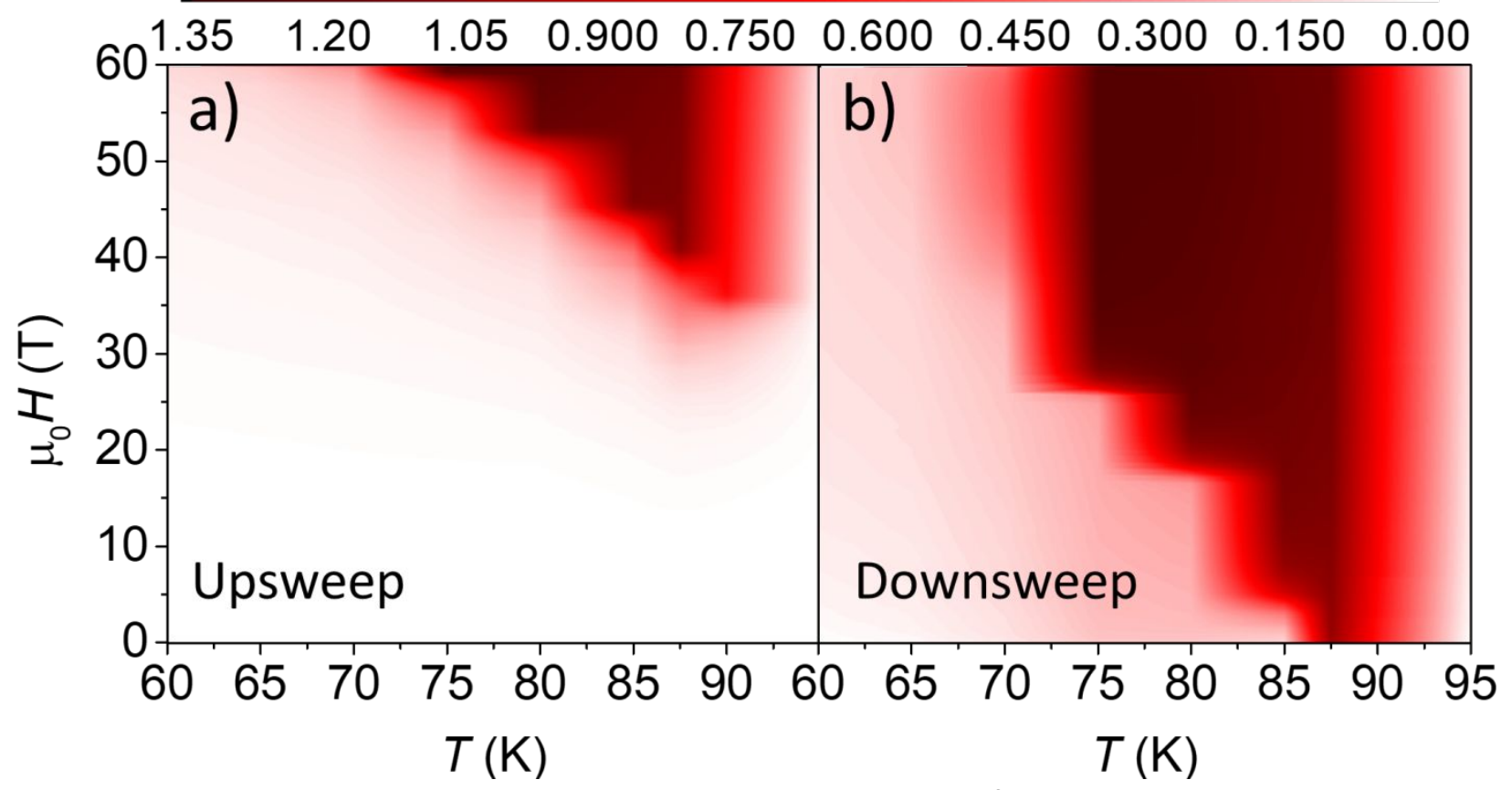

Figure S18 Electric polarization $\Delta P$ vs temperature $T$ and magnetic field $\mu_{0} H$ in a colour plot, measured in millisecond pulsed fields for up and downsweeps of the field.

\section{S5 References}

1. Turner, M. J.; McKinnon, J. J.; Wolff, S. K.; Grimwood, D. J.; Spackman, P. R.; Jayatilaka, D.; Spackman, M. A. CrystalExplorer 17.5, University of Western Australia: 2017.

2. Carpenter, M. A.; Salje, E. K. H.; Graeme-Barber, A., Spontaneous strain as a determinant of thermodynamic properties for phase transitions in minerals. Eur. J. Mineral. 1998, 10 (4), 621-691.

3. Salje, E. K. H.; Wruck, B.; Thomas, H., Order-parameter saturation and low-temperature extension of Landau theory. Z. Phys. B: Condens. Matter 1991, 82 (3), 399-404.

4. Carpenter, M. A.; Meyer, H.-W.; Sondergeld, P.; Marion, S.; Knight, K. S., Spontaneous strain variations through the low temperature phase transitions of deuterated lawsonite. Am. Mineral. 2003, 88 (4), 534-546. 